أ/ هبة سمير سليمان محمود الجندى

الثورة الصناعية الرابعة ومتطلبات تحقيقها في الجامعات المصرية

أ/ هبة سمير سليمان محمود

معيدة بقسم أصول التربية

كلية التربية جامعة عين شمس أفرل

تحت اشر افت جامعة

ا.د احمد محمد محمد عبد العزيز

استاذ أصول التربية

كلية التربية جامعة عين شمس
ا.د صفاء احمد محمد شحاته

استاذ أصول التربية

كلية التربية جامعة عين شمس التربية

د. مروة محمد سمير الموجي

مدرس أصول التربية

كلية التربية- جامعة عين شول التربية

مقدمة

في ظل وجود تغير ات متسار عة، يقف العالم اليوم على أعتاب ثورة صناعية رابعـة،

لا يقتصـر أثرهـا على تغييـر شـكل الصـناعات وطـرق الإنتـاج كمـا حدث فـى الثـورة الصناعية الثالثة، بل يمتد إلى تغيير فى المعرفة من حيـث الانتـاج والاسـتخدام و التطـوير الرقمى، مما يتطلب مـن مؤسسـات المجتمـع بصـفة عامـة و المؤسسـات التعليميـة بصـفة خاصة تلبية متطلبات تلك الثورة بما يسمح للمجتمع بأن يكون عنصر اً فعالاً مشـاركاً فى تللك الثورة بكافة مجالاتها. هذا ومرت الثورة الصناعية بالعديد من المراحل، ولقد كان لكل مرحلة من هذه المر احل ملامحها الخاصة وتداعيتها، و انعكاساتها على المجتمعات وقطاعاتها المختلفة، وأهم ما يميز الثورة الحالية أنها تستند إلى الثورة الرقمية أي الثورة الصناعية الثالثة، كما ترتكز على الرقمنة الشاملة لجميع الأصول المادية والتكامل في النظم الإيكولوجية الرقمية مع شركاء سلسلة القيمة، فهي تربط بين مجموعة واسعة من التقنيات الرقمية الجديدة حيث تتميز بشبكة الإنترنت الأكثر انتشارًا في كل مكان، أجهزة استشعار أصغر 
الثورة الصناعية الرابعة ومتطلبات تحقيقها في الجامعات المصرية

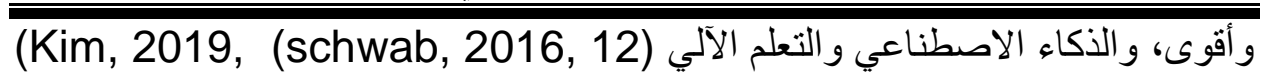

(Jadhav \& Mahadeokar, 2019, 106) 4)

وفي هذا الصدد فإنه مع ظهور هذه التقنيات النانشئة، وبروز اقتصاد المعرفة،

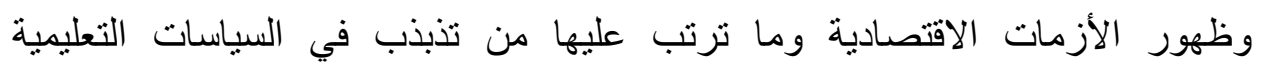
وسياسات تمويل الجامعات وغيرها من الأزمات قد فرض على على مؤسسات التعليم الجامعي العديد من الدنطلبات الرئيسية حتى تواكب هذه المؤسيسات عصر الثوات الثورة

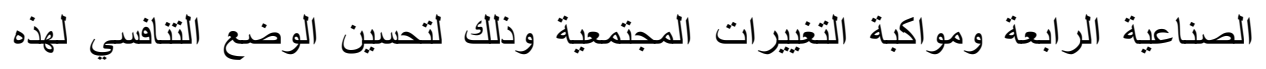

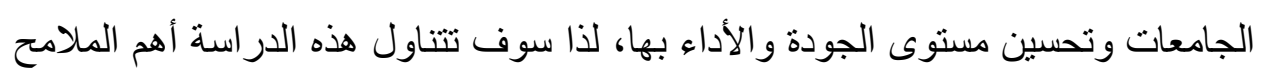
الرئيسية لهذه الثورة ومنطلباتها الأساسية من الجامعات.

الاراسات السابقة:

عرضت الدراسة عدداً من الدراسات السابقة المنعلقة بالثورة الصناعية الرابعة

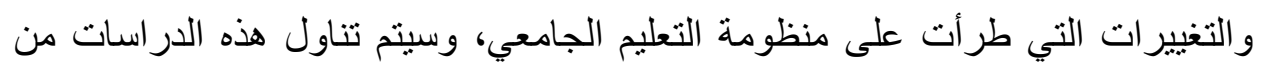

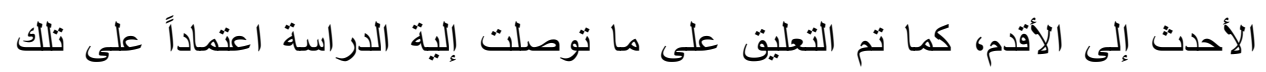

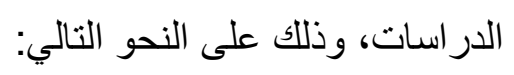

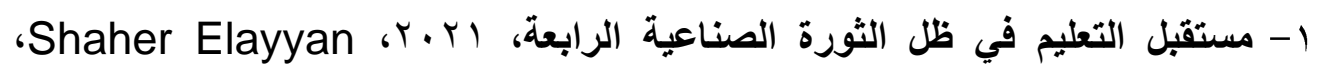
عمان. تهذف هذه الدراسة إلى زيادة الوعي المجتمعي حول آثار الثورة الصناعية

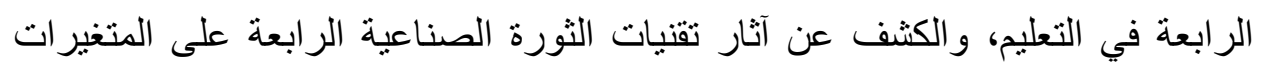
التعليمية كالتحكم في التعلم، وفرص التعلم، والأنشطة التعليمية، والآثار الاجتماعية. واقترحت الدراسة تتفيذ بعض التحولات الهامة في البرامج التعليمية والهناهج وبيئة

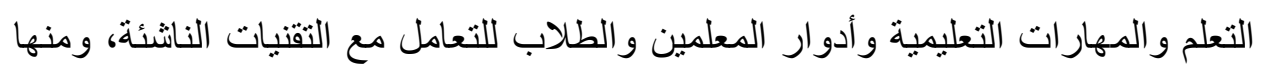
التكامل بين التخصصات كما هو الحال في نهج العلوم والتكنولوجيا والهندسة والرياضيات وكذلك التكامل بين عناصر النظام التعليمي (السياسة التعليمية وأعضاء هيئة التدريس و المناهج وبيئة التعلم و الطلاب) و القطاعات الصناعية في المجتمع، علاوة النطاكية 
أ/ هبة سمير سليمان محمود الجندى

على ضرورة اكساب الطلاب بعض المهارات الأساسية كالمهارات التقنية و التفكير النقدي والتنسيق مع الآخرين والاتصالات اللفظية و إدارة الوقت.

ץ- الابتكارات في التدريس والتعلم: استكشاف تصورات قطاع التعليم حول الثورة Adekunle Oke \& Fatima Araujo Pereira ، الصناعية الرابعة (IR (Y)

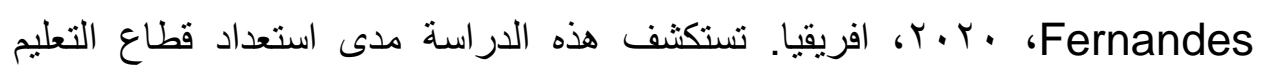
للثورة الصناعية الرابعة بقارة افريقبا، واعتمدت هذه الدراسة على المقابلات شبه

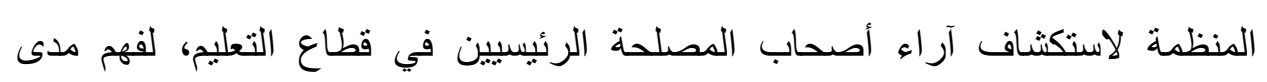

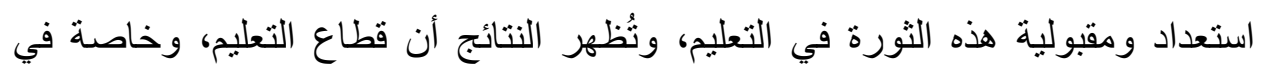

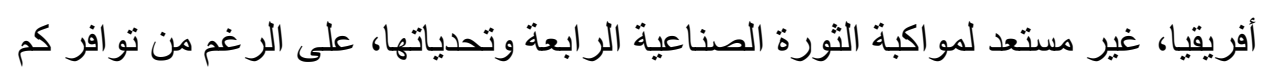
هائل من الامكانات والموارد التي تساعد على التقدم، كما قدمت هذه الدراسة التحديات

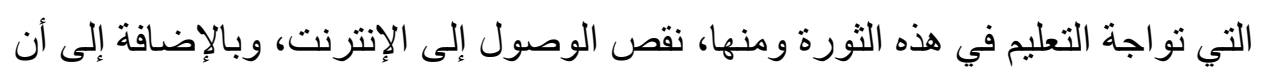

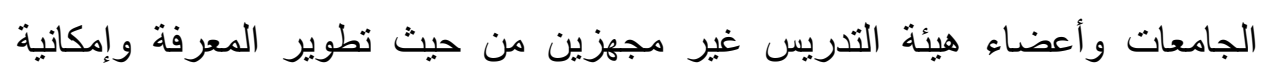
استخدام الابتكارات المتاحة، بالإضافة إلى أن المناهج الدراسية لا تنتوافق مع التغييرات

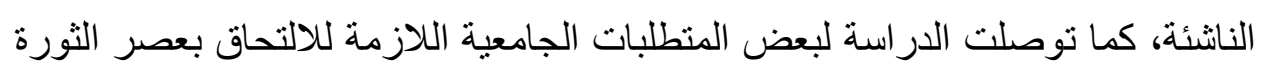

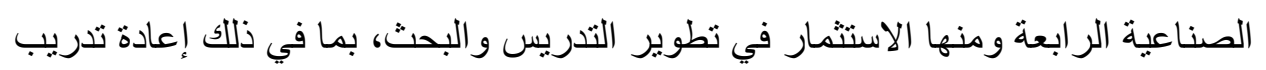

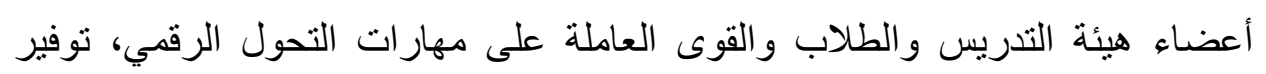
الأدوات والتقنيات المناسبة للتحول الرقمي، والتركيز على شراكة الجامعات مع ولئه المؤسسات التنموية.

ب- تصور مقترح للانتقال بالجامعات المصرية إلى جامعات الجيل الرابع في ضوء الثورة

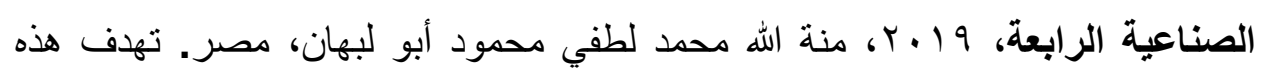

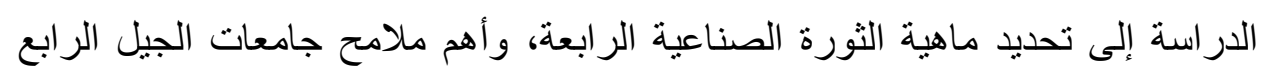
و الوقوف على اهم المتطلبات اللازمة للتحول إلى جامعة من الجيل الرايع، بالإضافة إلى

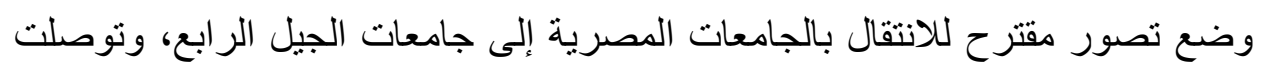

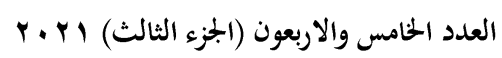

جلة كلية التربية- جامعة عين شثس 


\section{الثورة الصناعية الرابعة ومتطلبات تحقيقها في الجامعات المصرية}

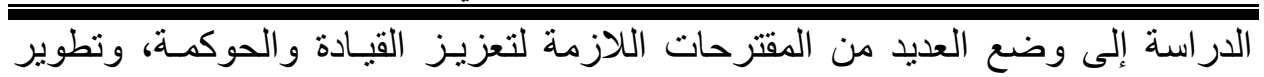
البرامج التعليميـة، والتدريس والتعلم والتقييم التقني، والبحث العلمي، والتدريب الوظيفي، والابتكار الذكي، والخدمات الذكية، و التدويل، و التعلم مدى الحياة. צ- الثورة الصناعية الرابعة: بناء المؤسسات الصناعية الرقمية، 7 أب ا، القمة العالمية للصناعة والتصنيع PWC \&(GMIS)، الامارات العربية المتحدة. يهدف هذا التقرير إلى التعرف على إطار الثورة الصناعية الرابعة ومراحل تطورها كما أشنار إلى وجود بعض المؤسسات في دول الخليج تعتمد على تقنيات الثورة الصناعية الرابعة، علاوة على أن الحكومات في الثرق الأوسط بدأت في استخدام هذه التقنيات الحديثة للقيام بالعديد الوظائف، ومن أبرز نتائجها أن تسهم بتسريع العولمة وتركز على الأفراد و الثقافة لدفع عجلة التقدم كما تحقق استثمار ات ضخمة ذات آثار و وعوائد سريعة. التعليق على الدراسات السابقة: أوضحت الدراسات السابقة تغيير شامل في شكل الجامعات التقليدية لتتحول إلى جامعات تقوم بتوظيف تكنولوجيا المعلومات والاتصالات والتقنيات الناشئة في الثورة الصناعية الرابعة كانترنت الأشياء، والذكاء الاصطناعي و غير ذللك، في العملية التعليمية وتعتمد على وسائل متقدمة تسمى هذه الجامعات بجامعات الجيل الرابع، وتعتمد أيضاً على تكامل جميع عناصر المنظومة الجامعية، كما تؤكد الدراسات السابقة على ضرورة تعزيز الثراكة بين المؤسسات الجامعية و المؤسسات التنموية، هذا و أقرت بعض هذه الدراسات على دور ها في تغيير هذه الثورة لسوق العمل المستقبلي مما يؤدي بطبيعة الحال لتغيير الوظائف المستقبلية ويتبعه تغيير في شكل القوى البشرية الملائمة ومهار اتهم اللازمة لذلك.

\section{قضية الدراسة وأسئلتها}

تمثلت قضية الدراسة في أن الثورة الصناعية الرابعة فرضت العديد من التحديات على المجتمعات ومؤسساته بصفة عامة والمؤسسات الجامعية بصفة خاصة، مما أدى إلى وجود تداعيات و انعكاسات غيرت من شكل وطبيعة الجامعات وبالتالي ظهرت 
أ/ هبة سمير سليمان محمود الجندى

متطلبات خاصة للجامعات في عصر الثورة الصناعية الرابعة، وقد صاغت الباحثة قضية الدر اسة في الأسئلة التالية:

( ) ما الملامح الأساسية للثورة الصناعية الر ابعة وأهم تداعياتها؟ r) ما التحديات التي فرضتها الثورة الصناعية الرابعة على الجامعات؟ ماته

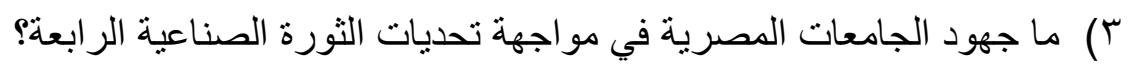
ع) ما متطلبات دخول الجامعات المصرية لعصر الثورة الصناعية الرابعة؟ أهداف الاراسة: تسعى الدر اسة الحالية إلى تحقيق الأهداف التالية: ( ) عرض وتحليل الثورة الصناعية الرابعة، من حيث المفهوم، والخصائص، ومدى الثى تأثير تقنبات الثورة الصناعية الرابعة على التعليم، بالإضافة إلى تداعيات الثورة الصناعية الر ابعة على الجامعات.

r) عرض أهم التحديات التي فرضنها الثورة الصناعية الرابعة على الجامعات. r) التعرف على واقع جهود الجامعات المصرية في عصر الثورة الصناعية الرابعة ومواجهة تحدياتها الرئيسة.

ء) التطرق لأهم المتطلبات الجامعية للالتحاق بعصر الثورة الصبناعية الر ابعة. أهمية الار اسة:

تتضح أهمية الدراسة الحالية في كونها تتعرض بالتحليل والمناقنة للأسس

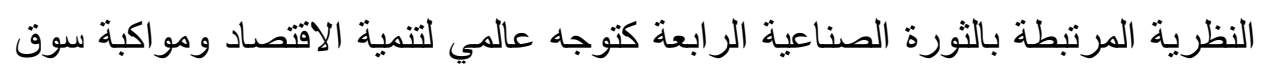
العمل، وللملامح المميزة للتعليم الجامعي المصري في ضوء الثورة الصناعية الرابعة، هذا لهابها إلي جانب ما سوف تتتاوله الدراسة من طبيعة الدور الذي تؤدية الجامعات في ظل متطلبات الثورة الصناعية الر ابعة وتداعياتها الجامعية.

\section{منهج الاراسة:}

نظراً لطبيعة الدراسة وأهمية تحليل ورصد ملامح الثورة الصناعية الرابعة، فقد اعتمدت الدراسة لتحقيق هذه الأهداف علي المنهج الوصفي، نظراً لقدرته علي تحليل 


\section{الثورة الصناعية الرابعة ومتطلبات تحقيقها في الجامعات المصرية}

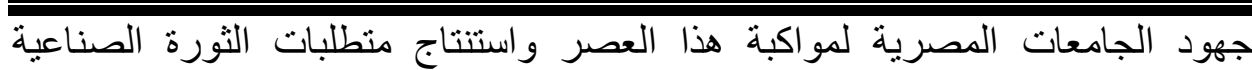

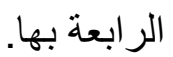

\section{المبحث الأول: الثورة الصناعية الرابعة وتداعياتها التعليمية}

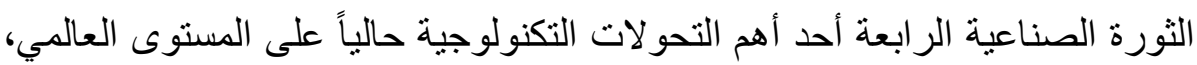

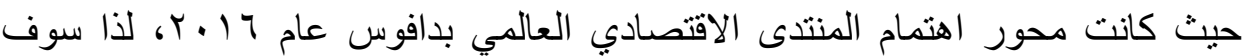

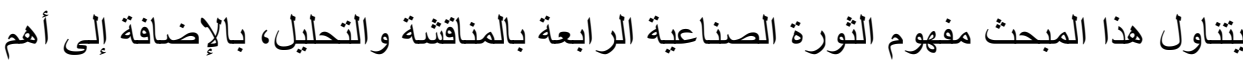

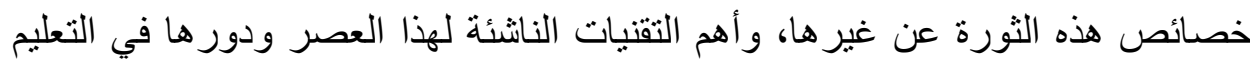
الجامعي، علاوة على تداعيات الثورة وانعكاساتها على الجامعات وذللك كالآتي: أولاً: مفهوم الثورة الصناعية الرابعة يحاول الجزء الحالي رصد مفهوم الثورة الصناعة الرابعة من خلال العديد من الجوانب ، وذلك من أجل إدراك أهمية مواكبتها على المستوى العالمي والإقليمي

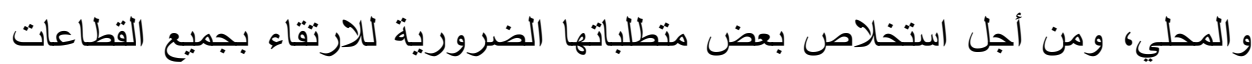

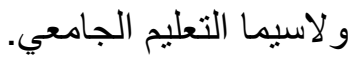
يرى رون ديفيد (Ron Davies) أن الثورة الصناعية الر ابعة هي مصطلح يطبق على مجموعة من التحولات السريعة في تصميم وتثتغيل وخدمة أنظمة التصنيع والمنتجات، و هي الخلف لثثلاث ثورات صناعية سابقة التي تسبيت في قفزات نوات نوعية في

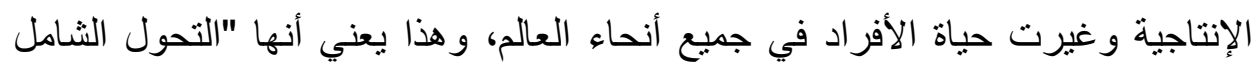

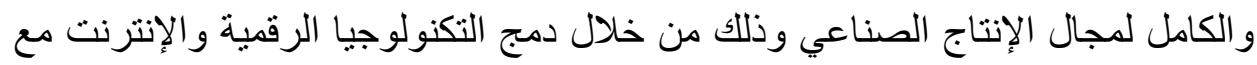
الصناعة التقليدية". (Davies, 2015, 2) ويعرفها جانك ساب يام (Jung-Sup_Um) بأنها "عملية تحويل نظام الإنتاج من خلال دمج عالم الإنترنت الذي تمثله تكنولوجيا المعلومات والعالم الحقيقي الذي كان موضوع الثورة الصناعية الأولى والثانية، أي أنها تربط العالم المادي (علية الإنتاج) بالعالم الإلكتروني (الإنترنت والكمبيوتر)". (Um, 2019, 3-4)

العدد الخامس والاربعون (الجزء الثالث) ا r. r r

جلة كلية التربية- جامعة عين شمس 
أ/ هبة سمير سليمان محمود الجندى

وتحليلاً لما سبق، يتضح أن المفاهيم السابقة ركزت على الأساس العلمي للثوة الصناعية الرابعة والذي يُعد "ربط العالم الحقيقي بالعالم الافتر اضي"، كما انطلقت من أن التحول الرقمي يقتصر على قطاع الصناعة، والذي بدوره يؤدي إلى نطور نظام

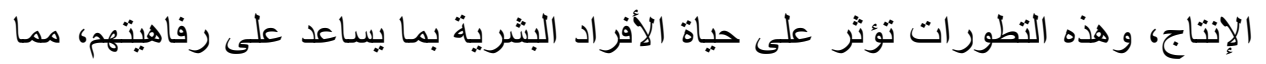

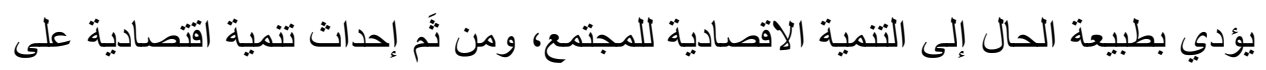
مستوى العالم، وذلك اعتمدت هذه المفاهيم على وجهة نظر اقتصادية فقط. وفي ضوء المفاهيم السابقة، وضعت الدراسة مفهوم للثورة الصناعية الرابعة و الذي وهي يتمثل في أنها موجة جديدة من التحولات التي تؤدي إلى حدوث تغيير في كافة الأنظمة

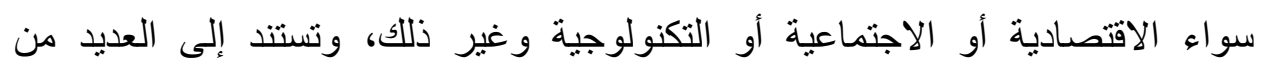
الابتكار ات التقنية ودمج العالم الحقيقي بالعالم الافتر اضي ,كما أنها تعتمد على العديد من التقنيات التي تتمثل في انترنت الأشياء، والذكاء الاصطناعي، الأنظمة الفيزيائية السيبر الية، والطباعة ثلاثية الأبعاد، و الواقع المعزز ، و غير ذلك.

\section{ثانياً: خصائص الثورة الصناعية الرابعة}

تتسم الثورة الصناعية الرابعة بالعديد من الخصائص لعل من أبرزها ما يلي:

(Blanchet et. al, 2014, 8, 9) (Krisnawati et. al, 2019, 53) (Y) (معاد، 19 (World Economic Forum b, 2017, 7) (Benešová \& Tupa, 2017,2195- 2196) (Koziol et. al, 2018, 3) (schwab,2016) () الرقمنة: أي استخدام تطبيقات التحول الرقمي في كافة المجالات، والانتقال بالخدمات إلى أعمال مبتكرة تعتمد على هذه التقنيات الناثئة، فهي أول ثورة صناعية

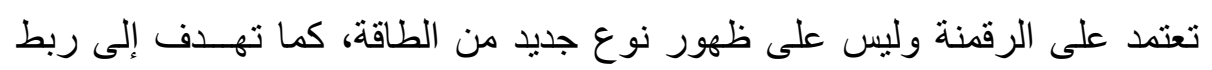
جميع وسـائل الإنتاج لتمكين تفاعلها في الوقت الفعلــي. 
الثورة الصناعية الرابعة ومنطلبات تحقيقها في الجامعات المصرية

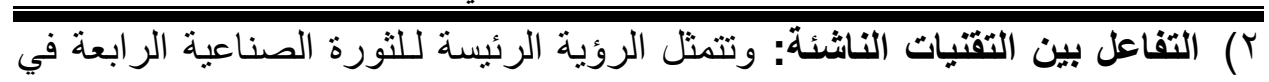
ربط التقنيات ببعضها البعض في كافة المجالات فعلى سبيل المثال، المصانع الذكية تقوم على أساس ربط مر افق الإنتاج بالأنظمة الفيزيائية السيبر الية، هذا بالإضافة إلى لى

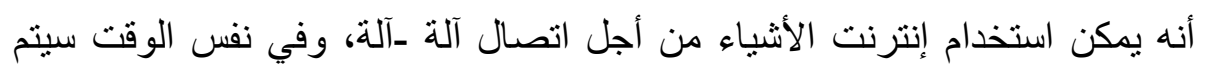
الحصول على كمية هائلة من البيانات، لهذا السبب أصبح من الضروري تحليل البيانات الكبيرة لتكون قادرًا على التنبؤ بالفشل المحتمل والتكيف في الوقت الفعلي مع الظروف المتغيرة. r) التغيير الإبداعي: حيث تؤدي التقنيات الجديدة والتفاعل بينهما إلى ظهور طرفًا جديدة للإبداع والاستهلاك، بالإضافة إلى تغيير طريقة تقديم الخدمات العامة

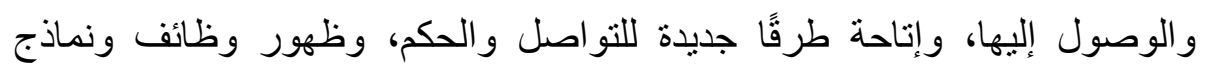

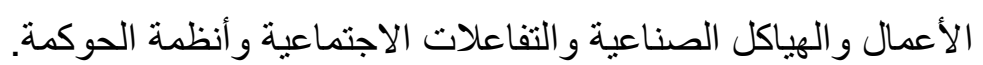
ع) السرعة: أي السرعة في إمكانية تطوير الابتكارات ونشرها؛ وهذا نتاج للعالم المتر ابط بشكل أعمق و إن التكنولوجيا الجديدة تولد تكنولوجيا أحدث و أكثر قدرة. ه) الاتساع والعمق: فالاعتماد على الثورة الرقمية يجمع بين تقنيات متعددة يؤدي إلى جئ تحو لات كبيرة في الاقتصاد والأعمال والكيفية التي نعمل بها بل وتؤثر على الإنسان كذلك. 7) التأثير والتعميم حيث إنها ستتضمن تحولاً في مختلف المجالات عبر كل الدول و المؤسسات والمجتمعات، فالتقنيات الناثشئة لها تأثثر كبير على كافة المجالات، ولن يتمكن من التحكم في هذه التقنيات سوى الموظفين المؤهلين وذوي التعليم العالي، لذا يجب أن تتعاون الجامعات مع المؤسسات الصناعية كافة.

\section{ثالثاً: تقنيات الثورة الصناعية الرابعة}

تتنوع تقنيات الثورة الصناعية الر ابعة وتتعدد مجالاتها وتتعاظم أهميتها على كافة

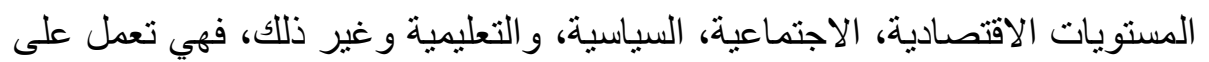


أ/ هبة سمير سليمان محمود الجندى

إعادة تشكيل المجتمع والسياسات والمؤسسات وأسواق العمل كما تسهم في تغيير أنماط و أساليب الانتاج و الاستهلاك. وفيما يلي عرض لبعض وذه هذه التقنيات التي أثرت بشكل فعال على التعليم الجامعي، وكان لها دور بارز في العملية التعليمية وغيرت

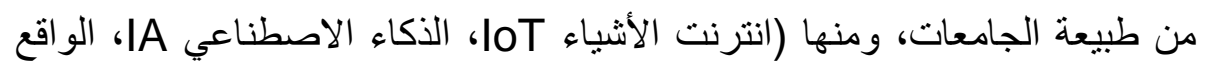
المعزز AR)، وذلك على النحو التالي:

\section{( ) إنترنت الأشياء IoT) Internet of Things):}

لقد أصبح مصطلح انترنت الأشياء من المفاهيم الثائعة والرائجة في الآونة الأخيرة، حيث يشير إلى امتلاك الأشياء المختلفة القدرة على التواصل ببعضهاء هـ التها البعض من خلال شبكة الانترنت لأداء وظائف معينة ومحددة.

حيث تعرفها منظمة ماكينزي Mckinsey على أنها أجهزة استشعار متصلة بواسطة الثبكات بأنظمة الكمبيوتر، يمكن لهذه الأنظمة مراقبة أو إدارة الآلات المتصلة، بالإضافة إلى إمكانية مراقبة العالم الطبيعي والأشخاص و الحيوانات. ويرى آخرون أنها "شبكة مفتوحة وشاملة من الأجززة الذكية التي لديها القدرة على التنظيم التلقائي، ومشاركة

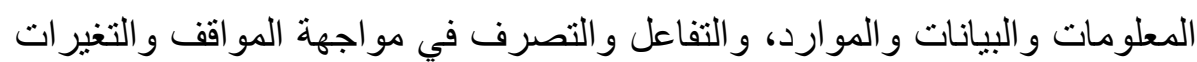
في البيئة". (Asghar et. al, 2020, 5) وذكرت بعض الأدبيات أن الفكرة الأساسية لـ (loT) تتمثل في وجود مجموعة متنوعة من الكائنات مثل أجهزة الاستشعار والمحركات القادرة على التفاعل مع بعضها

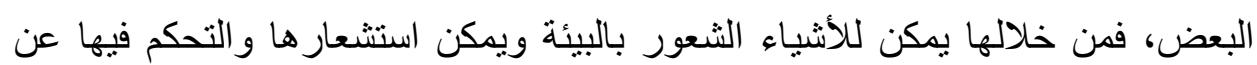
(Abbasy \& Quesada, بُعد من خلال البنية التحتية للشبكة الحالية مثل الإنترنت 2017, 914) وتنكل تقنياتها الأساسية بالتعليم الجامعي في اللوحات البيضاء الذكية والوسائط الرقمية التفاعلية الأخرى التي يمكنها جمع وتحليل البيانات لأعضاء هيئة التدريس 
الثورة الصناعية الرابعة ومنطلبات تحقيقها في الجامعات المصرية

والطلاب لتحسين التدريس وتحسين نتائج التعلم، بالإضافة إلى أجهزة استشعار درجة الحرارة الذكية ومعدات التدفئة والتهوية وتكييف الهواء الذكية التي تقلل من استهلاك الطاقة وأتمتة إدارة العمليات داخل المحاضرة، وبطاقات هوية الطلاب الذكية، وأجهزة تتبع الحضور، و أقفال الأبواب اللاسلكية وكاميرات المراقبة المتصلة وأنظمة التعرف

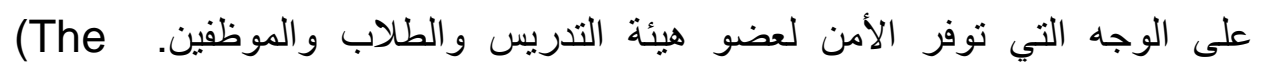
Alcatel-Lucen, 2020, 2) كما تتمتع تقنية إنترنت الأشياء المتقدمة بفو ائد كبيرة على التعليم العالي، ولهذا السبب يجب دمجها في الدورات الأساسية لـ STEM (العلوم والتكنولوجيا والهندسة و الرياضيات) والتعليم والتدريب المهني، كما أطلقت هذه التقنية العنان لتقنيات الهاتف المحمول، هذا ويؤدي انتشار الأجهزة المحمولة إلى التعلم القائم على الألعاب، مما يساعد على زيادة مشاركة الطلاب وتحفيز هم على إكمال المهام، وتعزيز التعاون، وتنشيط الروح التنافسية وتعزيز كفاءاتهم الرقمية. (Grinshkun \& Osipovskaya, 2020)

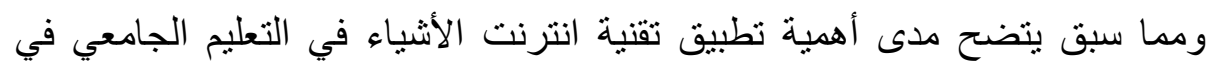
الوقت الحالي، وذلك نتيجة تميز ها بالمرونة في العملية التعليمية حيث تتمتع بإمكانية

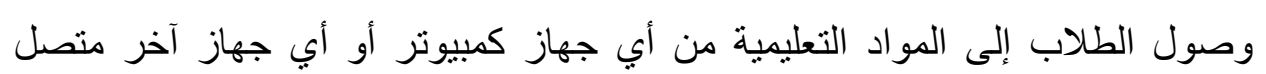

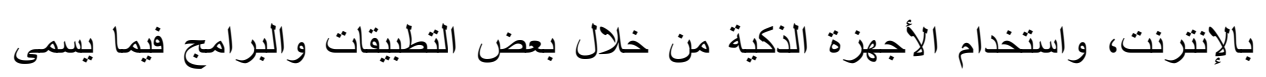
بالفصول الافتراضية، هذا بالإضافة إلى أنه يوفر نظاماً تعليمياً أكثر جاذبية عن طريق وليق

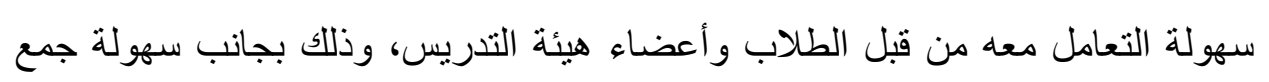
وتحليل وتخزين البيانات في شبكة الانترنت، ولكن هذا يعرض خصوصن هية الطالب للخطر ، ويعرضها للاختر اق. 
أ/ هبة سمير سليمان محمود الجندى

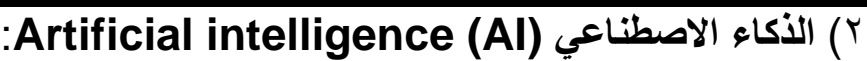

يُعد الذكاء الاصطناعي واحداً من أهم تقنيات الثورة الصناعية الرابعة لذا تعددت المفاهيم الخاصة بهذه التقنية وفيما يلي عرض لبعض هذه المفاهيم، وأهم تطبيقاتها في التعليم الجامعي.

يمكن تعريفه على أنه مصطلح لأنظمة الكمبيوتر التي بمكنها الثعور ببيئتها، و التفكير، والتعلم، و التصرف استجابة لما يشعرون به في ضوء أهدافهم المبرمجة. (Herweijer \& Waughray, 2018, 3) حل المشكلات المعقدة بعقلانية أو اتخاذ الإجراءات المناسبة لتحقيق أهدافه في أي ظروف واقعية يواجها. The National Science and Technology Council (NSTC), The Office of Science and Technology Policy (OSTP), 2016, 6) ومن أكثر تطبيقاته رواجاً هو "التعلم الآلي" Machine learning (ML)، الذي يعني اكتساب الأجهزة الذكية للمعرفة عبر معالجة كميات هائلة من البيانات للتعرّف على الأنماط المتكررة وتحديد العلاقات المشتركة وتطبيق القواعد ومن ثم استشعار الخلل و التعامل معه. (دائرة الثؤون الخارجية والاتصالات وشركة تنمية نفط عُمان ش م م،

حيث تستخدم الجامعات خوارزميات التعلم الآلي لقبول الطلاب أو رفضهم، كما يمكن لأنظمة الذكاء الاصطناعي تقييم مستويات الطلاب، وتوجيههم خلال الدورة التدريبية بناءً على الذكاء الجماعي جنبًا إلى جنب مع الخبرة الفردية، وتقييم نتائج الاختبار تلقائيًا وحتى أتمتة التفاعل بين الطالب والمعلم باستخدام روبوتات المحادثة وتقنيات البرمجة اللغوية العصبية. The United Nations Educational, Scientific and Cultural Organization, 2019, 33) 
الثورة الصناعية الرابعة ومتطلبات تحقيقها في الجامعات المصرية

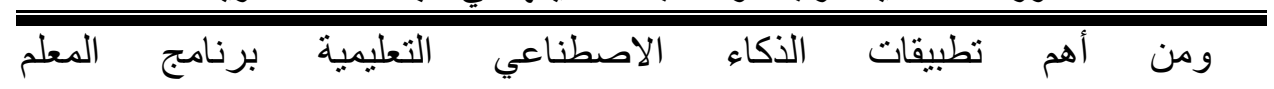
الآلي(Teacherbots) الذي يعد بمثابة واجهة خوارزمية معقدة، فهو يوفر المحتوى؛ كما أنه سيكون أيضًا بمثابة إثر اف أو توجيه للطلاب، هذا ويشير Teacherbots إلى إنى أي نوع من الأجهزة أو البرامج القائمة على الآلة، والتي يمكن أن تؤدي دور المعلم

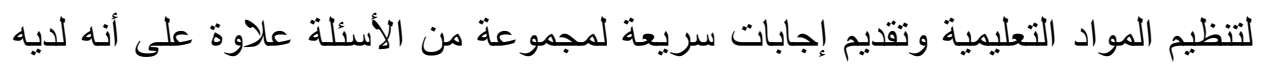
القدرة لتسهيل ومراقبة وتقييم وإدارة تعلم الطلاب أثناء التعلم عبر الإنترنت.

(Fahimirad \& Kotamjani, 2018, 113)

ومما سبق يتضح أنه يمكن للذكاء الاصطناعي القيام بالمهام الميكانيكية الروتينية المتكررة كتصحيح ورصد الدرجات وتحليلها للاستفادة من المخرجات في تتمية الطلاب، و إعداد أنشطة مختلفة لهم، بالإضافة إلى إنشاء منصات ذكية متكاملة، مما يمنح للطلاب وأعضاء هيئة التدريس وقتاً للابداع و الابتكار و التفاعل مع بعضهم البعض في إلباء الجو انب الأكثر إبداعاً جنباً إلى جنب مع الذكاء الاصطناعي، حيث أنه لا يمكن الاستغناء

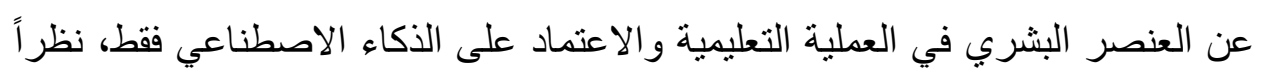

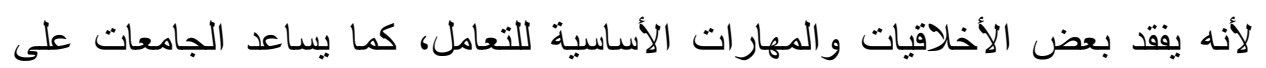
التنبؤ بالاحتياجات المستقبلية وتحسين جودة مخرجاتها التعليمية.

\section{(r) الواقع المعزز: Augmented Reality (AR)}

يُعد الواقع المعزز من المفاهيم الأساسية في عصر الثورة الصناعية الرابعة، لذا سوف يستكثف هذا القسم الاستخدامات الحالية للواقع المعزز في مجال التعليم لتحديد كيفية استخدامه حاليًا.

ويمكن تعريف الواقع المعزز على أنه مجال متنامي للتكنولوجيا حيث ينم تعديل الحياة الواقعية وتعزيزها من خلال المرئيات والأصوات الناتجة عن الكمبيوتر، كما يمكن استخدام الواقع المعزز في العديد من التقنيات المختلفة، مثل: أجهزة الكمبيوتر والأجهزة اللوحية والهواتف الذكية، ويتم استخدام تقنية الواقع المعزز من خلال 
أ/ هبة سمير سليمان محمود الجندى

المكونات القابلة للارتداء، منل النظار ات والخوذات، هذا ويسمح هذا الواقع المعزز بدمج الو اقع الافتر اضي القائم على المعلومات و الو اقع المادي للعالم الحقيقي.

(Sural, 2017, 200)

يعرفه آخرون على أنه "تقنية تستخدم الواقع الافتراضي لإضافة كائنات افتراضية

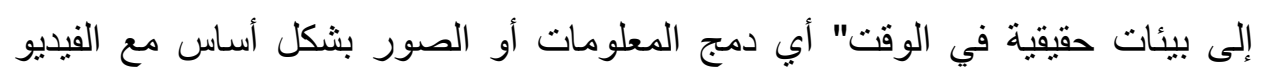
المتدفق من كاميرا الويب. (Munnerley et. al, 2014, 25) هذا وتتمحور الفكرة الرئيسة للواقع المعزز في إقناع المتعلم بأن البيئتين، الواقعية و الظاهرية، تتعايشان معاً.

(Rizov \& Rizova, 2015, 8)

تكمن التطبيقات التعليمية بالجامعات لهذه التقنية في بيئات التعلم الافتراضية لنقل المعرفة والمهار ات بصورة متطورة، والتي تتم من خلال تنفيذ الواقع (VLEs)

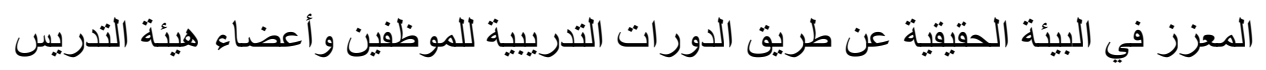
و الطلاب الجدد باستخدام نظارات الواقع المعزز، بالإضافة إلى دورها في تحويل

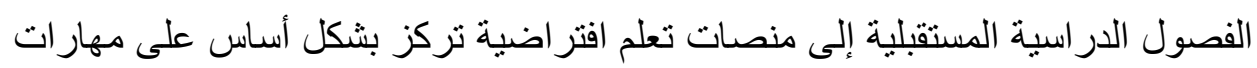
حل المشكلات والقدرة على التفكير النقدي والقيادة والتواصل الفعال و الأساليب المبتكرة في التعلم. , (Benešová \& Tupa, 2017)

2196)

ومن أهم تقنيات الواقع المعزز في التعليم هي الكتب المدمجة، فيمكن الطلاب قراءة الكتاب باستخدام نظام الو اقع المعزز الذي يعرض المشاهد على الصفحات المادية التي نم

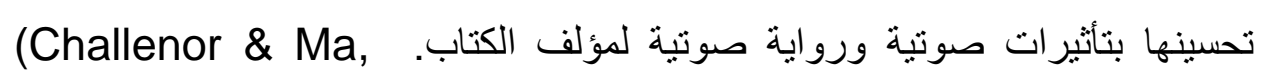

$2019,2)$

من خلال العرض السابق لتقنيات الثورة الصناعية الرابعة نستتتج أن هذه التقنيات الحديثة تُعد أداة تسمح بشرح المحتوى الدراسي وتوفير معلومات إضافية للطلاب بجانب المنهجية التعليمية المناسبة، وبذلك فهي تساعد على انتشار نموذج نعليمي تعاوني تفاعلي

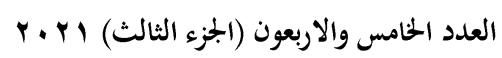

(175)

جلة كلية التربية- جامعة عين شمس 


\section{الثورة الصناعية الرابعة ومنطلبات تحقيقها في الجامعات المصرية}

ذاتي يساعد على زيادة معارف ومهارات الطلاب في مجتمع التعلم الرقمي، ومن هنا يوجد دور للجامعات في المساعدة على انتشار هذه التقنيات وتزويد الطلاب بالمهارات اللازمة لهذا العصر والمناسبة لمواكبة سوق العمل المستقبلي، وتغيير دور عضو هيئة التدريس وكذلك آليات تدريسه، وبالتالي فهي تقوم بتغيير شكل القوي البشرية اللازمة للوظائف المستقبلية. ررابعاً: تداعيات الثورة الصناعية الرابعة على الجامعات

تتنوع تداعيات الثورة الصناعية الرابعة وتقنياتها الناشئة لتشمل كافة القطاعات الصناعية والاقتصادية والتعليمية والصحية والسياسية وغير ذلك، ومن ثم يهدف هذا الجزء من الدراسة إلى رصد أهم هذه التداعيات على القطاع التعليمي ولاسيما الجامعات، وسوف يتناول هذا الجزء هذه التداعيات في النقاط التالية: الفصول الدراسية المعكوسة أو الفصول المقلوبة وهي طريقة تتكون من عكس طبيعة الأنشطة في الفصل الدراسي (المحاضرات) وفي المنزل (مهام الواجبات المنزلية)، و الذي يعني إعطاء الطلاب أنشطة مستقلة من المستوى المعرفي المنخفض يجب القيام بها في المنزل، من أجل تفضيل العمل التعاوني ومهام التعلم ذات المستوى المعرفي العالي في الفصل، من خلال وضع الطلاب في نشاط تعاوني. Gueye \&)

Exposito, 2020)

r) تغيير محتوى المناهج الأساسية في العديد من المجالات الأكاديمية، حيث أصبحت اليوم تركز على المواهب والمهارات الصعبة كالمخاطرة وغير ها من المهارات، كما أصبح من الضروري للجامعات اعتماد مناهج تربوية جديدة لتقديم استجابات مصممة خصيصًا للاحتياجات المحددة لكل متعلم، ولتسهيل الوصول إلى المحتوى التعليمي وتحسين جودة (Gueye \& Exposito, 2020) (World Economic Forum, التعلم. 2016, 20) 
أ/ هبة سمير سليمان محمود الجندى

هذا وتتضمن هذه المناهج علم أصول التدريس النشط الذي يشير إلى طرق التدريس التي تركز على الطالب والأنشطة التي يقودها المعلم، فهو نهج تربوي يهدف إلى جعل المتعلم ممثلاً في عملية التعلم الخاصة به مع المشاركة النشطة في بناء المعرفة. هذا بالإضافة إلى الاعتماد على التعلم القائم على المشروعات العملية والذي يعني ممارسة للتربية النشطة التي تسمح بإدارة التعلم من خلال تحقيق مشروع فردي أو جماعي. (Gueye \& Exposito, 2020) (World Economic Forum, 2016, 20) r) فقدان الكتب المدرسية وأعضاء هيئة التدريس دور هما كونهما مصدر أساس للمعرفة، كما يتم نقل دور أعضاء هيئة التدريس في المستقبل القريب إلى دور افتراضي، واستبدال المكتبات التقليدية بقو اعد بيانات المعرفة في تتسيقات الوسائط، بالإضافة إلى لى تزايد دور بيئات الألعاب والواقع المعزز في الأنشطة التعليمية، وهذا يتطلب إنشاء

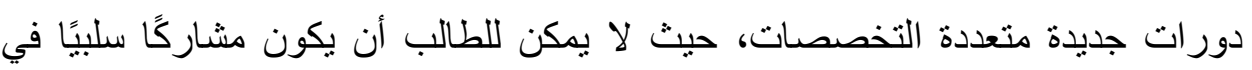
العملية التعليمية. (Sakhapov \& Absalyamova, 2018, 3§) التعلم المدمج، الذي يعد برنامجًا نعليميًا رسميًا يتعلم فيه الطالب جزئيًا على الأقل من خلال تقديم المحتوى والتعليم عبر الإنترنت مع بعض عناصر تحكم الطالب بمرور

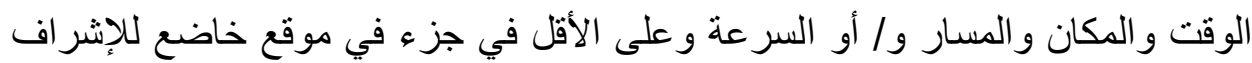

من قبل عضو هيئة التدريس. (Bryan \& Volchenkova, 2018, 24-25) فهو مفهوم هجين يجمع بين التدريس المباشر وجهاً لوجه عبر الإنترنت، ويدمج استخدام نظريات التعلم وممارسات التدريس في إعادة تصميم مرنة ومتعددة الوسائط ومتعددة الخطوط، يعتمد هذا النهج الذي تم تطويره على دمج الأنظمة الفيزيائية

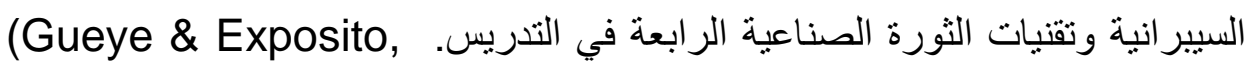

2020)

ه) ربط كل شيء عبر الإنترنت من البرامج الأكاديمية وطرق التدريس والمناهج الدراسية و غير ها، وبذلك يتم تغيير آليات التعلم وتلقي المعلومات من المعلم والمتعلم، على سبيل 
الثورة الصناعية الرابعة ومتطلبات تحقيقها في الجامعات المصرية

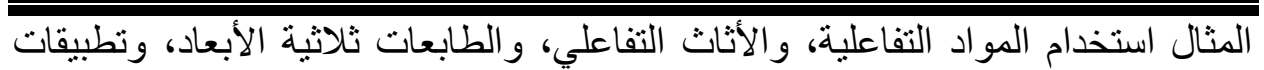
الهاتف الجوال. (Thang \& Dung, 2018, 171) 7) ظهور جامعات الجيل الرابع: حيث يظهر شكل جديد من الجامعة يقوم بالتنريس والبحث

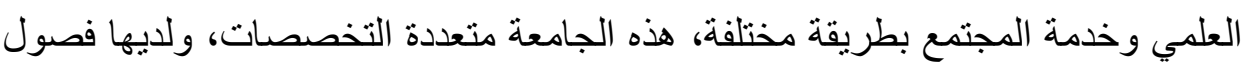

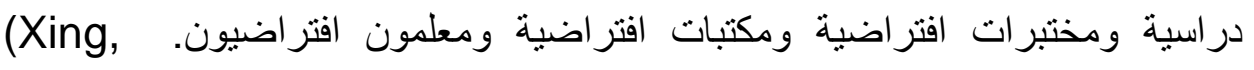
Marwala, 2017) تعد جامعات الجيل الرابع بيئة مفتوحة ومركزاً لمجموعة من الاتصالات و الأعمال

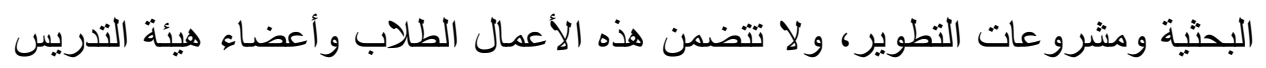

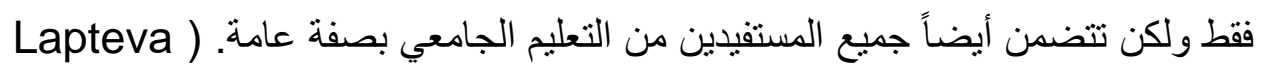
(\& Efimov, 2016, 2692

يتضمن واقع جامعات الجيل الرابع في تكوين أسس الفكر (الفئات، الدفاهيم، النماذج

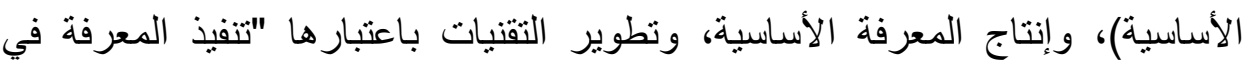

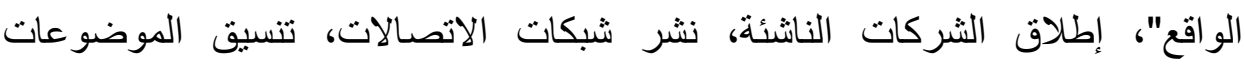
المختلفة، الكثف عن ممارسات جديدة، بالإضافة إلى الفصول الدراسية والمكتبات

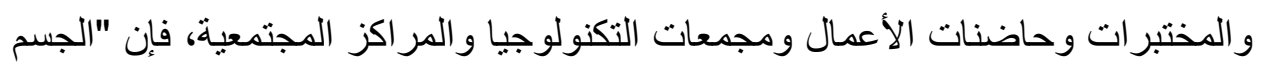

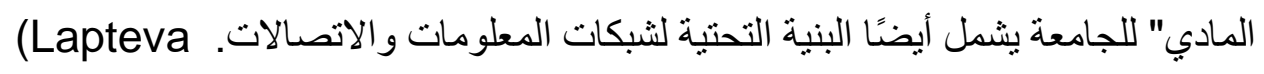
\& Efimov, 2016, 2692) ومما سبق يتضح أن هذه الجامعات تعتد في المقام الأول على التعليم الافتراضي من

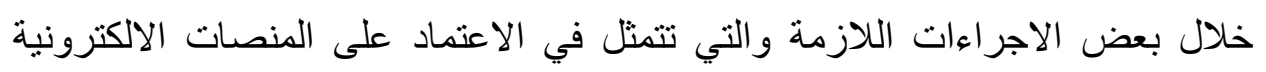

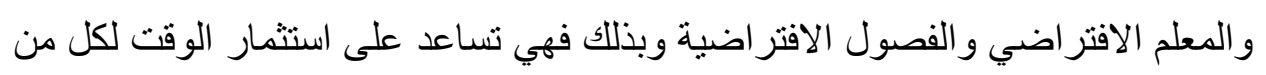

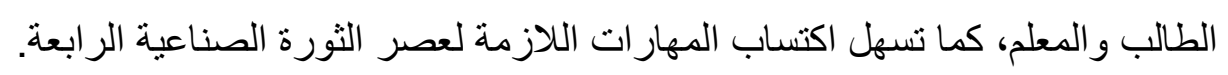

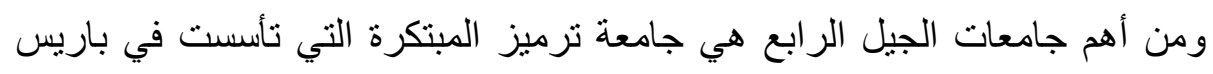

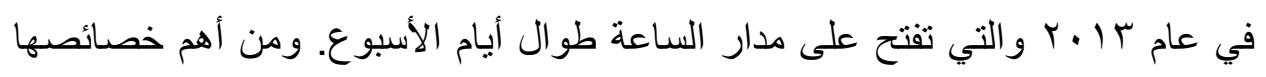

العدد الخامس والاربعون (الجزء الثالث) Y. r. T

جلة كلية التربية- جامعة عين شمس 
أ/ هبة سمير سليمان محمود الجندى

أنه لا يوجد مدرس أو كتب أو رسوم التعليم، كما يعمل الطلاب حسب مشروعاتهم ويخضعون لعدة برامج للتنريب الداخلي. وبمجرد الانتهاء من هذه المشروعات، سوف تكسب نقاط للانتقال إلى المستوى التالي. (Shahroom \& Hussin, 2018, 315, 316) ونستتنج مما سبق أن شكل التعليم الجامعي تغير ليصبح قائم على التعليم الرقمي، الذي يعني دمج المزيد من التقنيات الناشئة في العملية التعليمية، ويصبح للتكنولوجيا دور اً رئيساً في التعليم، هذا ويهدف التعليم في عصر الثورة الصناعية الرابعة إلى بناء جيل قادر على بلى مواكبة التغييرات المتسارعة، مما أدى إلى تغيير الدور الرئيس لعضو هيئة التدريس،

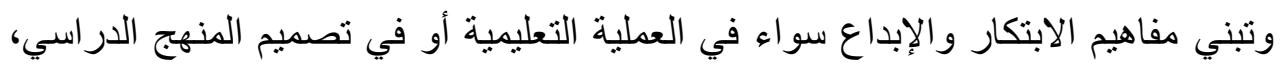
وبذلك يصبح التعليم أيضًا أكثر فاعلية نظرًا لمو اكبته لمتطلبات الجيل الحالي. المبحث الثاني: تحديات الثورة الصناعية الرابعة

وفي إطار التعرف علي ملامح الثورة الصناعية الرابعة يحاول الجزء الحالي رصد أهم التحديات التي تفرضها الثورة الصناعية الرابعة علي المؤسسات بصفة عامة و الجامعات بصفة خاصة، وذلك من أجل تحليل جهود الجامعات المصرية في ضوء هذه التحديات، وأهم متطلبات الجامعات المصرية للتكيف مع تحديات الثورة الصناعية الرابعة، وذللك علي النحو التالي:

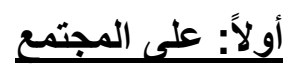

وتقوم الدر اسة برصد أهم هذه التحديات في النقاط النالية: Common Fund)

(Federal Ministry (Quyet, 2020, 423) for Commodities, 2018, 1- 2) (Manda \& Dhaou, 2019, of Labour and Social Affairs, 2017, 95) (Carter, (Xu et. al, 2018, 92: 94) (Formunyam, 2019, 280) 247) (World Economic Forum b, (schwab, 2016, 47, 48) 2019,18- 20) (World Economic Forum a, 2017, 1) 2017, 10) (1) وجود فجوة تكنولوجية جديدة يمكن أن تزيد من تقييد القدرة التنافسية وتؤثر على التطلعات المنز ايدة للبلدان النامية المعتمدة على السلع الأساسية تنمية مستدامة. 
الثورة الصناعية الرابعة ومنطلبات تحقيقها في الجامعات المصرية

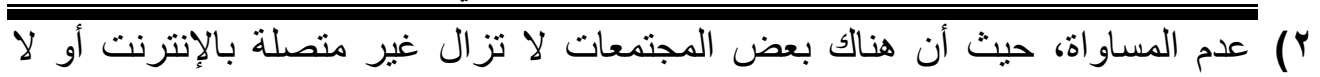
تسنطيع الوصول إلى التقنيات الجديدة، بالإضافة إلى عدم المساواة في توزيع الدخل، وما ينتج عنه من تحمل تكاليف التقنيات الجديدة لأغر اض تعليمية. r) خصوصية البيانات حيث تثير المخاوف المتعلقة بالخصوصية والأمان في التكنولوجيا مشكلات التقة في العصر "الذكي"، حيث يتم إنشاء البيانات الضخمة اليوم بأحجام كبيرة عبر الاتصال بالإنترنت وتخزينها، وهذا يجعل من السهل إدخال البيانات من الثبكة

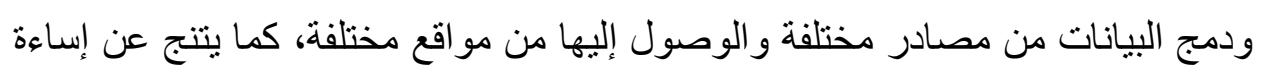
استخدام البيانات بشكل فعال من التجسس الصناعي أو الهجمات التي يقوم بها المتسللون أو التخريب.

؛ ) المخاوف الأخلاقية؛ حيث أصبحت الروبوتات المدعمة بالذكاء الاصطناعى وقدرة التعلم

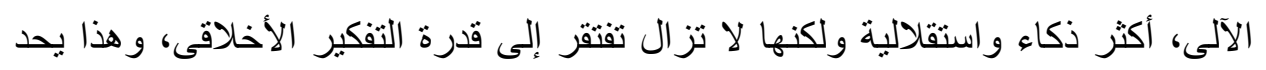

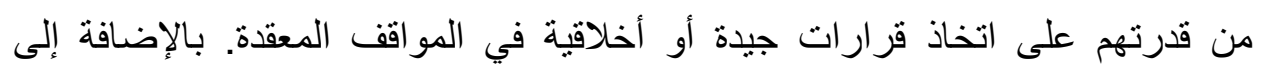

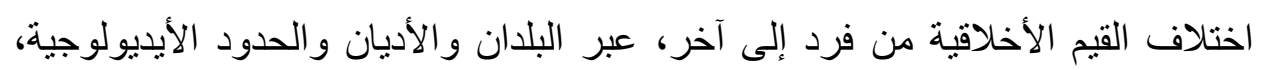

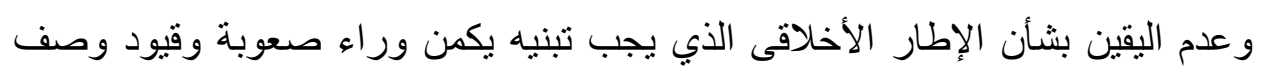

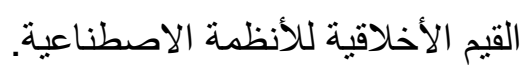

•) يمكن للتكنولوجيات الجديدة مثل الطائرات بدون طيار، و التعرف على الوجه، والتحليلات السلوكية أن تضع التقنيات الجديدة والمدمرة في أيدي الأنظمة القمعية الفاسدة.

T) محدودية فهم المؤسسات المختلفة لطبيعة التغييرات المدمرة، والمواءمة ضئيلة أو معدومة بين استراتيجيات القوى العاملة واستراتيجيات ابتكار الثركات، وقيود الموارد وضغوط الربحية قصيرة الأجل. نتيجة لذلك، هناك عدم تطابق بين حجم التغييرات القادمة والإجر اءات الهامشية نسبيًا التي تتخذها المؤسسات لمواجهة هذه التحديات. 
أ/ هبة سمير سليمان محمود الجندى

( اختفاء بعض الوظائف منخفضة المهارة و المتكررة، هذا بالإضافة إلى وظائف الخدمات، ومن المرجح أن تزيد البطالة، وهذا يمكن أن يؤدي إلى أعداد أكبر من المهاجرين الاقتصاديين ويتبعه بطبيعة الحال زيادة في عدم المساو اة. ^) عدم التطابق بين المهار ات الحالية والمؤهلات المطلوبة لوظائف المستقبل، كما أن حوالي

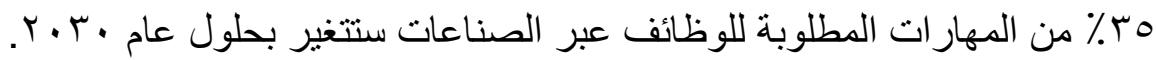
ثُانياً: على الجامعات

وتتمحور هذه التحديات فيما يلي: (Cox, (Nkosi et. al, 2020, 2117-2118) (Quyet, 2020, 422) (Satpathy et. al, 2020, 2) 2018, 4) (Lawrence (Manda \& Dhaou, 2019, 247) (Ignatowski, 2017, 5) (Formunyam, 2019, 279) et. al, 2019, 517) (1) نقص البنية التحتية من الكوادر المدربة سواء عضو هيئة تدريس أو الإدارة أو القيادات الجامعية أو طلاب. r) معظم الجامعات غير قادرة على إنتاج خريجين جاهزين للعمل دون أن تكتسب الأوساط الأكاديمية فهمًا و اضحًا لما تبحث عنه المؤسسات في أصحاب العمل المبتدئين. r) سعر أجهزة الكمبيونر قد يكون متاح لبعض الطلاب لدفع ثمنها، ومُبالغ في ثمنه بالنسبة

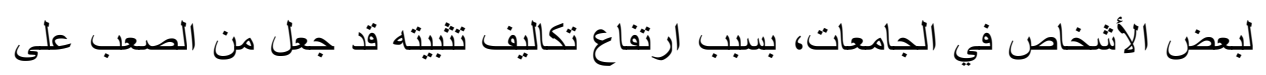
الجامعات في البلدان النامية تحقيق وتوصيل وسائل لتكنولوجيا المعلومات والاتصالات للمحاضرين و الطلاب لتكون قادرة على استخدامها. §) لا تزال العلاقة بين الجامعات والمؤسسات التنموية والبحث العلمي وأنشطة نقل التكنولوجيا ضعيفة. ه) وجود فجوة بين المهارات المطلوبة اليوم وتلك المطلوبة في المستقبل، ونتيجة لذلك، تميل عروض المؤسسات التعليمية إلى التخلف عن الاقتصاد الحقيقي. 


\section{الثورة الصناعية الرابعة ومتطلبات تحقيقها في الجامعات المصرية}

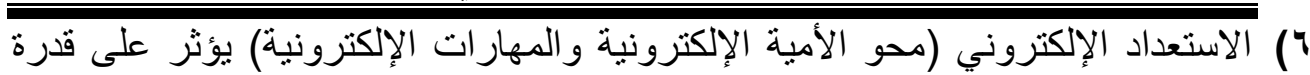
المواطنين على المشاركة الكاملة في الأنشطة الاجتماعية والاقتصادية في المجتمع الإنه الذكي، حيث يوجد انخفاض في مستويات الاستعداد الإلكتروني في معظم الدول وخاصةً

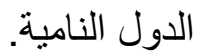

V ندرة وجود ربط للأهداف التعليمية الخاصة بالبرامج التدرييية بهدف إيجاد وظائف للمتعلمين؛ بالإضافة إلى ضعف الربط بين معايير التعليم العالي المحلية والدولية. ^) وجود نوع من أعضاء هيئة التدريس ممن لديهم المعرفة بالتكنولوجيا ولكنهم برفضون استخدامها في الفصول الدراسية بسبب غياب وجود خطط للقيام بذلك. 9) بعض أعضاء هيئة التدريس غير مقتنعين بالمزايا المحتملة للتقنيات الجديدة فيما يتعلق بدعم عملية التدريس/ التعلم التي تظهر بطريقة ما مقاومة على مستوى أعلى. • 1) يشعر بعض أعضاء هيئة التدريس أنهم ليسوا خبراء في التعامل مع التكنولوجيا ويحتاجون إلى مزيد من الوقت الكافي للتعلم نظرًا لأن تعلم التقنيات و البرامج الجديدة يستغرق وقتًا طويلاً. (1) إعادة صياغة مناهج التدريس والتخصصات التربوية في الجامعات بما يتناسب مع منطلبات الثورة الصناعية الرابعة، لذلك ستضطر الجامعات باستمرار إلى تحديث مناهجها الدراسية حتى تتمكن من التعامل مع تحديات الأمن السيبر الى للمؤسسات لكي بلهي

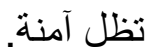
ץ (1) صعوبة التوصل إلى مناهج متكاملة تتضمن كل تقنيات الثورة الصناعية الرابعة ومتطلباتها، هذا بالإضافة إلى الحاجة لأنماط جديدة من التدريس. ب ا) اللجوء للمناهج البديلة التي تستجيب للاحتياجات المجتمعية المتغيرة باستمرار لتكون السمة المميزة للتعليم في هذه الثورة. 


\section{أ/ هبة سمير سليمان محمود الجندى}

\section{المبحث الثالث: واقع جهود الجامعات المصرية في عصر الثورة الصناعية الرابعة}

خطت مصر العديد من الخطوات نحو مواجهة تحديات الثورة الصناعية الرابعة وتلبية متطلباتها، وسوف تستعرض الدراسة في هذا الجزء الجهود المصرية التي تساعد على تعزيز التعليم الذي يتناسب مع متطلبات الثورة الصناعية الرابعة من قبل الدولة و الجامعات المصرية لمواكبة عصر الثور الصناعية الرابعة، وذللك على النحو التالي:

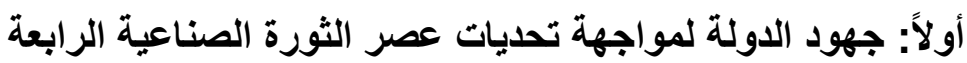
قامت الدولة بالعديد من المبادرات والإجراءات اللازمة لمواجهة تحديات الثورة الصناعية الرابعة وتلبية متطلباتها داخل الجامعات المصرية. انطلاقاً من محاولة الدولة لسد فجوات الاحتياجات المستقبلية لسوق العمل في

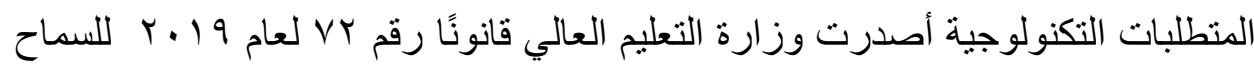
بإنشاء جامعات تكنولوجية لأول مرة في مصر، وتهدف هذه الجامعات إلى تطوير دورات جديدة متكاملة للتعليم والتدريب التكنولوجي التطبيقي بالتوازي مع مسار التعليم

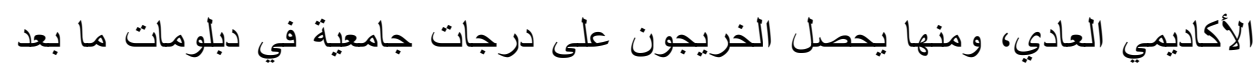

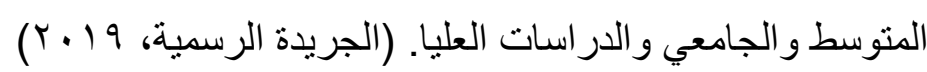
و استجابة لهذا القانون، وضعت وزارة التعليم العالي والبحث العلمي خطة موحدة

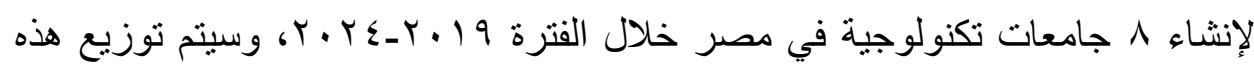
الجامعات على أساس التوازن الجغرافي لضمان التغطية الوطنية وستغطي جميع

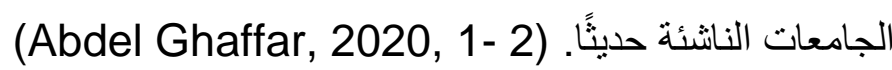
كما قامت الحكومات المصرية بالبدء في إنشاء (آ) جامعات تكنولوجية جديدة هي جامعات: (شرق بورسعيد- 7 أكتوبر - برج العرب- الأقصر الجديدة (طيبة)- أسيوط - بابهـ سمنود بمحافظة الغربية)، وذلك بخلاف (r) جامعات سبق إنشاؤها وبدأت الدراسة بها هي جامعات: (القاهرة الجديدة - قويسنا - بني سويف) التكنولوجية، و البدء في إنشاء الجامعة المصرية لتكنولوجيا المعلومات بالعاصمة الإدارية الجديدة بالتعاون مع وزارة 
الثورة الصناعية الرابعة ومنطلبات تحقيقها في الجامعات المصرية

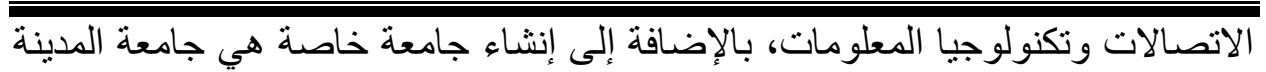

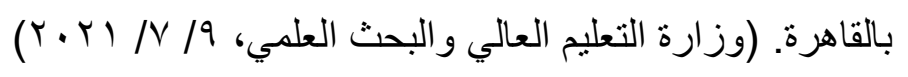

علاوة على استراتيجية للعلوم والتكنولوجيا والابتكار التي وضعتها وزارة التعليم العالي والبحث العلمي في مصر التي تعزز وتدعم البحث والتطوير والابتكار في التقنيات الناثنئة التي تساهم في تحديات المستقبل، بالإضافة إلى إنشاء ب مر اكز امتباز رئيسة في الجامعات للبحث والتطوير في مجال الطاقة المتجددة وإدارة موارد المياه

والتكنولوجيا الحيوية. (Abdel Ghaffar, 2020, ولتعزيز البحث والابتكار للتقنيات النانشئة والتحول السريع وضعت مصر صندوق البحث والتطوير ليقارب 1\% من الناتج المحلي الإجمالي، حيث يمثل هذا الصندوق بيئة مواتية لتطوير أبحاث ومنتجات جديدة تعتمد على تحليل البيانات الضخمة والتعلم الآلي، و التكنولوجيا الحيوية، وتكنولوجيا النانو، و الطاقة المتجددة، والروبوتات، و إنترنت

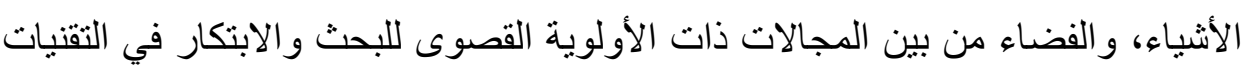

الناشئة. (Abdel Ghaffar, 2020, 3 ) ومن ضمن جهود الدولة التي تدعم الابتكار معرض القاهرة الدولي للابتكار كسوق

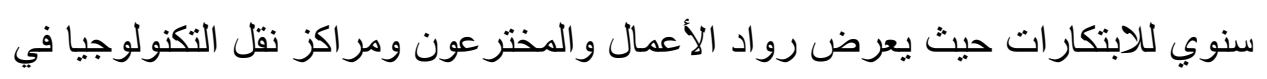
الجامعات والمعاهد ومراكز البحث وطلاب الجامعات ورعاية المبتكرين وشركات

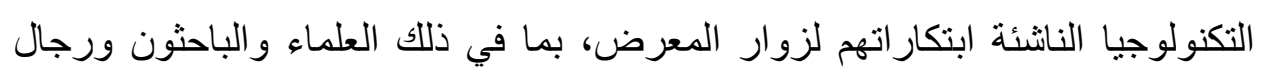
الأعمال و الصناعة والاستثمار وتمويل البحث العلمي. (Abdel Ghaffar, 2020, 6)

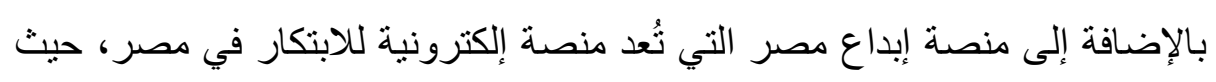
تهدف إلى تسليط الضوء على المبتكرين المصريين محليًا و عالميًا، و إيجاد نقاط التقاء بينهم، وبناء جمعيات تعاونية بين ممثلي بيئة الابتكار وريادة الأعمال المصرية. بالإضافة إلى ذلك، تهدف المنصة إلى تثقيف المبتكرين و الثركات الناشئة عن إدارة

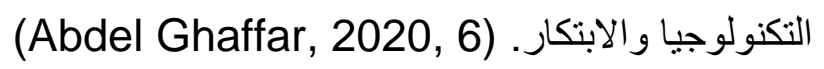


أ/ هبة سمير سليمان محمود الجندى

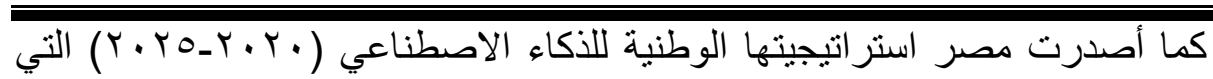
تهدف إلى إطلاق العنان للقدرات المحتملة للذكاء الاصطناعي في مجالات مختلفة كالتعليم، والبحث و التطوير، و الرعاية الصحية، و النقل، والثورة الصناعية الرابعة، و الزراعة الدقيقة، والخدمات المالية، وغير ذلك. ولتحقيق هذه الاستراتيجية، أنشأت مصر المجلس القومي للذكاء الاصطناعي وفقًا لقرار رئيس مجلس الوزراء لمتابعة و التحكم في تتفيذ الاستر اتيجية من خلال التتسيق بين الجهات ذات العلاقة. كما اهتمت وزارة التعليم العالي والبحث العلمي بإعداد جيل متخصص في الذكاء الاصطناعي وتطبيقاته، لذلك تم إنشاء كليتين جديدتين متخصصنتين في الذكاء الاصطناعي، كما تم إضافة قسم للأكاء الاصطناعي إلى بعض كليات الحاسبات و علوم المعلومات. Abdel

(Ghaffar, 2020, 9)

هذا بالإضافة إلى دور أكاديميـة البحث العلمي والتكنولوجيـا عندما قامت بإطلاق البرنامـج القومسى للحاضنـات التكنولوجيـة في أكتوبر 10 ـ ب بحيث يصبـح أكبر مظلة لإنشاء و إدارة الحاضنات التكنولوجية في منظومة ريادة الأعمال والابتكار بحيث تغطي أقاليم مصر المختلفة، وأن تكون قادرة على تحويل الأفكار والابتكار ات ومخرجات

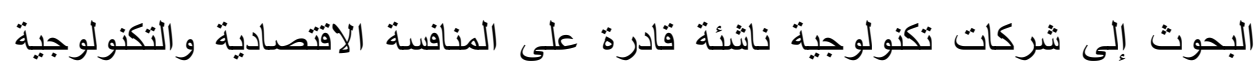

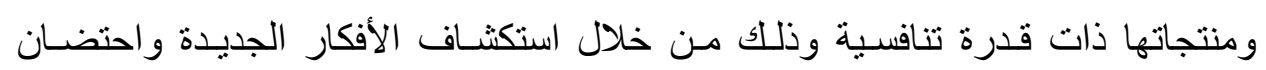
أصحابها مـن الباحثين والمبتكرين ورواد الأعمال والطلاب فهى الجامعات المصرية

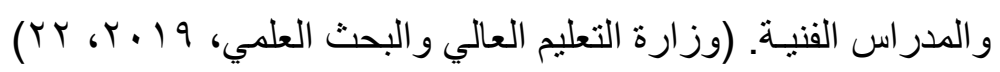
و هذه الحاضنات متخصصسة في مجـال النسيج والذكاء الصناعى و انترنت الأشياء

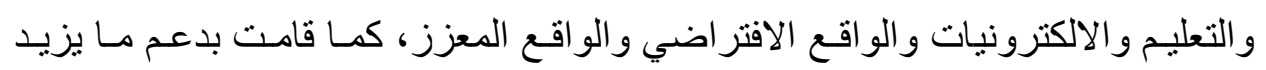
عن ·و شـركة تكنولوجيـة وتخريـج عدد با شـركة عاملـة في الأسواق المحلية او

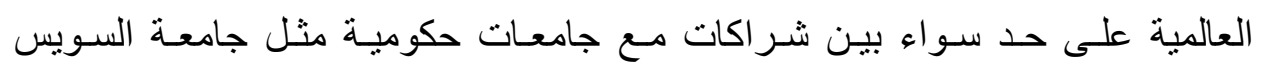

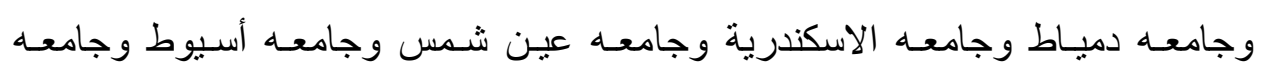




\section{الثورة الصناعية الرابعة ومتطلبات تحقيقها في الجامعات المصرية}

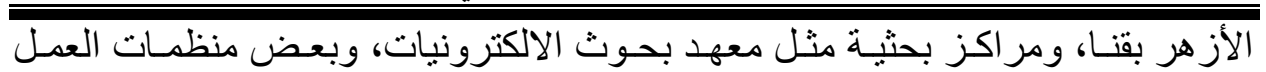
الددني مثل مؤسسـة مصر الخير ومؤسسـة اتصال، وبالتعاون مع جهات أجنبية عاملة في مجال دعم الحاضنات والابتكار وريادة الأعمال. (وزارة التعليم العالي والبحث

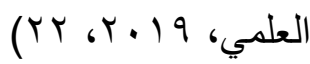

\section{ثانياً: جهود الجامعات لمواجهة تحديات عصر الثورة الصناعية الرابعة}

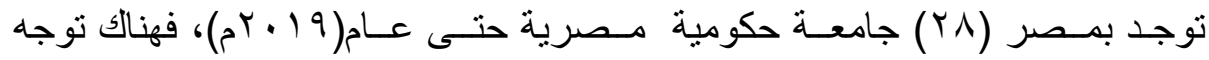

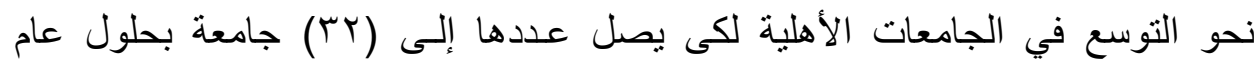

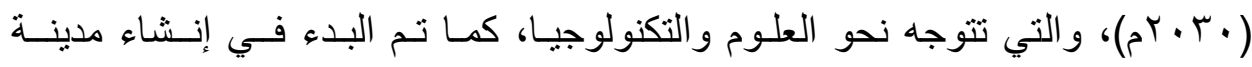

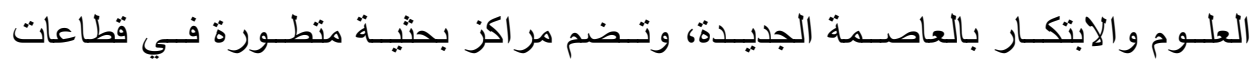

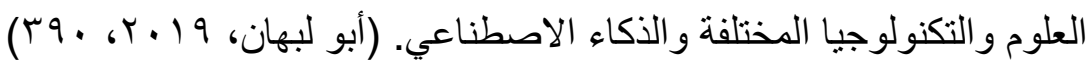

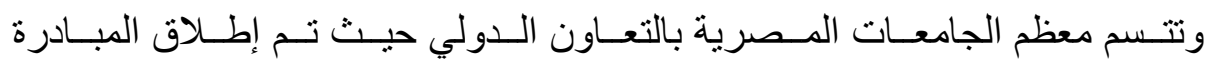

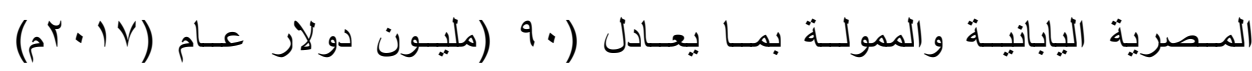
بهـدف تتميـة المـوارد البشرية في قطـاعي الصحة والتعليم، وتم إطلاق مبادرات

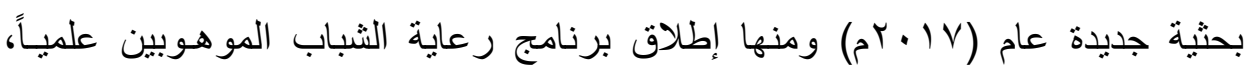

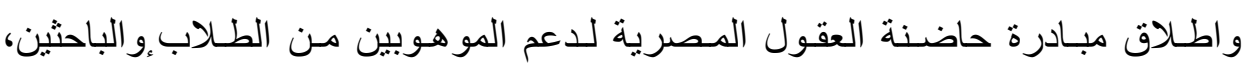

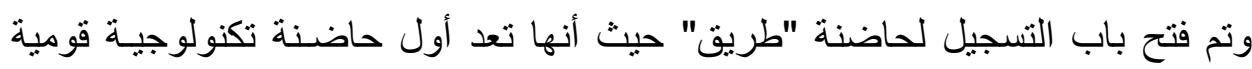

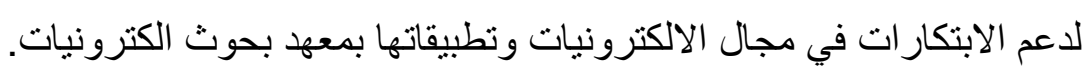

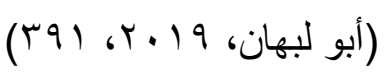

بالإضافة إلى افتتاح 00 مكتبًا لاعم الابتكار ونقل التكنولوجيا وتسويقها في الجامعات

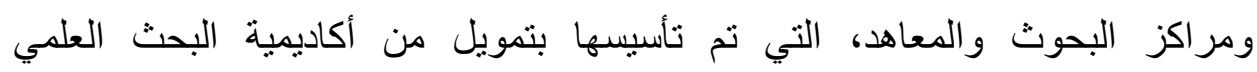
و التكنولوجيا، وتهذف هذه المكاتب إلى تفعيل دور البحث العلمي وربطه بالصناعة ودعم

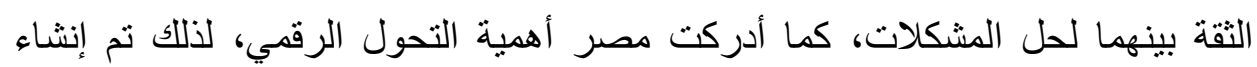




\section{أر هبة سمير سليمان محمود الجندى}

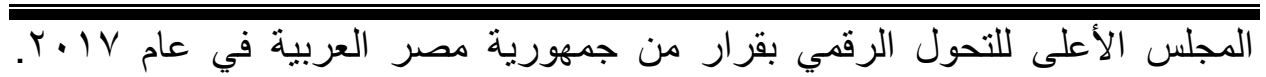

(Abdel Ghaffar, 2020, 6)

كما كانت هناك العديد من الإجراءات التي قامت بها الجامعات المصرية لتلبية منطلبات الثورة الصناعية الرابعة ومنها إستحداث صيغ جديدة لإنتاج معلم الألفية الثالثة

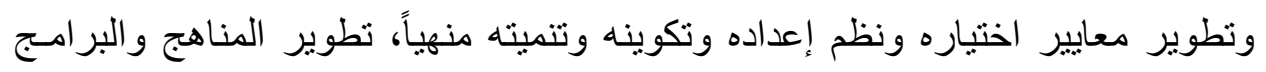

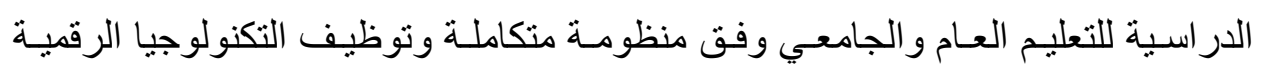

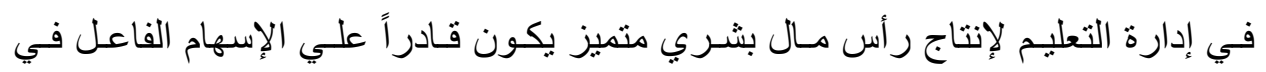
التنميـة المستدامة وبنـاء مجتمع المعرفة، ربط التعليم بقضايا التنمية البشرية التئية

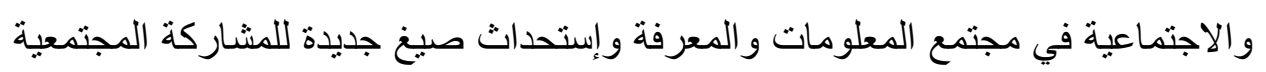

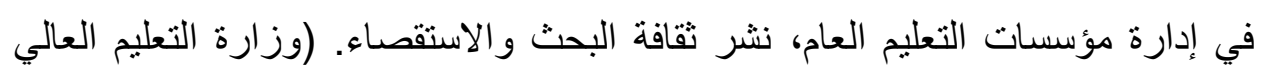

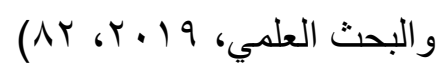

علاوة على إكساب الطالب مهارات البحث العلمي والابتكار دمج ذوي الإعاقة تعليميا

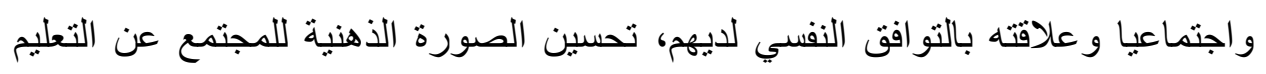

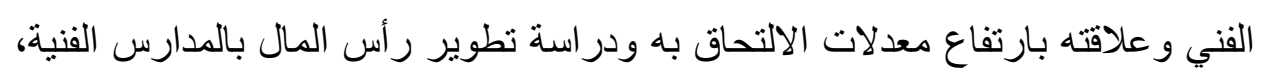

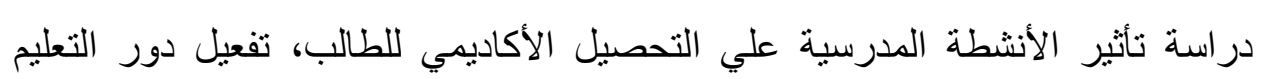

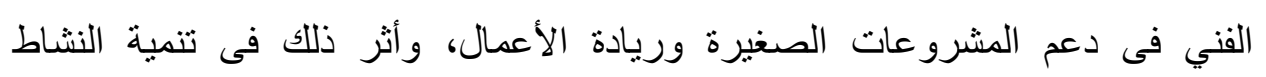
الاقتصاد المصري، دراسة دور التربية الوطنية للمتعلمين في تحسين الوعي البئئي

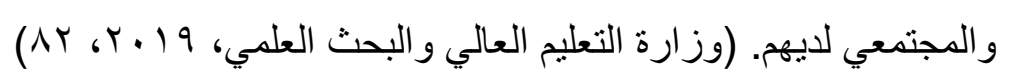

ويثير مـاسبق إلى أن الجامعات المصرية تسعى نحو التميز البحثي والتعـاون

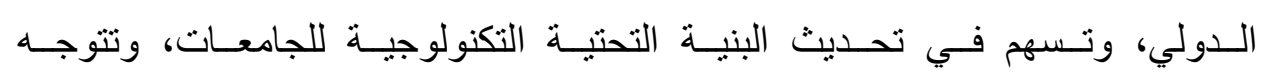

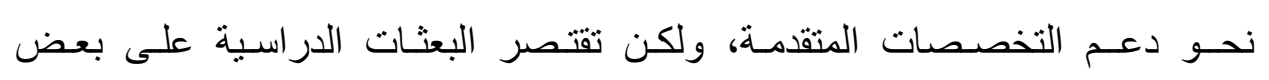

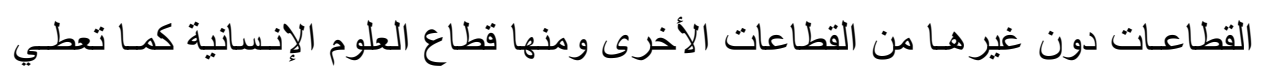

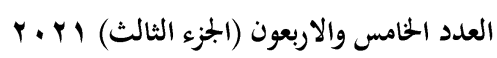
مجلة كلية التربية- جامعة عين شثس 
الثورة الصناعية الرابعة ومنطلبات تحقيقها في الجامعات المصرية

الأولويـة لتطوير تكنولوجيـا المعلومات والاتصالات بالجامعات لتطوير مجتمع المعرفة

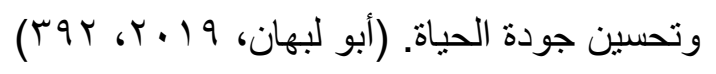

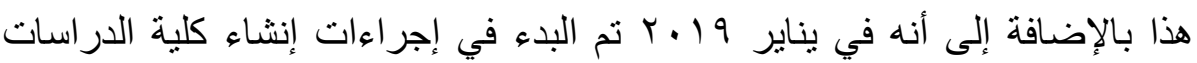
العليا للنانو تكنولوجي بجامعة القاهرة، لتكون أول كلية في مصر و العالم العربي والثرق الأوسط في مجال علوم النانو تكنولوجي، كما تم إعداد التصور الأكاديمي واللائحة الخاصة بالكلية وبر امجها الدر اسية، ودر اسة ما يتعلق بكافة مقومات إنشائها من امكانيات مادية وبشرية، وبدأت الدراسة في الكلية بعد حصول الجامعة على موافقة مجلس ولس الوزراء؛ واعتماد اللائحة الأكاديمية والهيكل الإداري والمالي للكلية من لجان المجلس بكس هوله

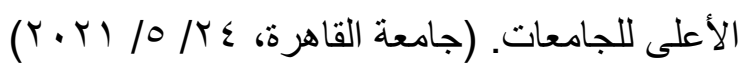

وحتى تتضح الرؤية نجد أن هدف هذه الكلية يتمثل في إعداد قاعدة عريضة من الباحثين و العاملين وتنشئة جيل من المبدعين و المجددين في هذا التخصص، واستخدام التعليم التكنولوجي في إجر اء البحوث المبتكرة التي تدعم معرفة واسعة وجديدة للطلاب واستخدام هذه المعرفة لتحقيق الفائدة المرجوة منها لصالح المجتمع. (جامعة القاهرة،

$$
(r \cdot Y) / 0 / r \varepsilon
$$

وفي ضوء هذا الهدف سعت الكلية إلى المساهمة في تشكيل مستقبل الصناعات المعتمدة على مواد النانو في مصر، من خلال إعداد نخبة من الباحثين الرواد القادرين على التعلم و الإبتكار واكتشاف مواد وتطبيقات جديدة في أسواق الصناعات المختلفة.

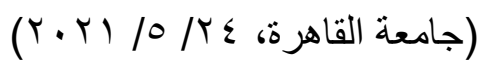

وتعد كلية النانو تكنولوجي الكلية الأولى من بين الكليات العملية بجامعة القاهرة التي تعتمد كليًا على الإدارة الرقمية لنظم المعلومات والمخازن والدراسات العليا، وتتضمن بر امج الكلية مجموعة من التخصصات هي: برنامج ماجستير العلوم في تقنيات التصنيع النانو تكنولوجي (هندسة النانو)، ويهدف إلى تأهيل المتقدم لدراسة علوم النانو في مجالات التطبيقات الهندسية والصناعية، وبرنامج ماجستير آخر في علوم النانو 
أ/ هبة سمير سليمان محمود الجندى

تكنولوجى الحيوية، ويهدف إلى تأهيل خريجين لدراسة تطبيقات تكنولوجيا النانو في مليمي المجالات الحيوية والطبية، بالإضافة إلى برنامجين للابلومات المهنية هما: برنامج دبلوم تصميم وتركيب أنظمة الطاقة الثمسية، وبرنامج دبلوم النانو تكنولوجي فى تقنيات البيئة

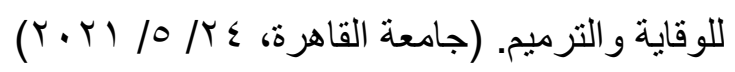
ومما سبق يتضح أن معظم الجامعات المصرية تقوم بالعديد من الاجراءات اللازمة لمواكبة الثورة الصناعية الرابعة ومنها، تحديث اللوائح السياسية والبرامج التعليمية و التدرييية لتتناسب مع منطلبات سوق العمل المستقبلية ومواكبة التوجهات العالمية في إطار التحول نحو جامعات الجيل الرابع، بالإضافة إلى ربط التعليم بتقنيات الثورة

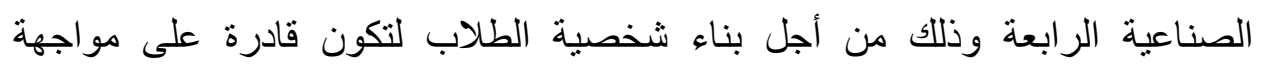
التغييرات الاقتصادية والاجتماعية المستقبلية، حيث قامت معظم الجامعات بإطلاق

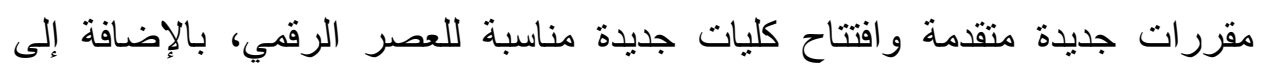
الاعنماد على التعليم المدمج والالكتروني و إطلاق منصات تعليمية في جميع الجامعات المصرية و إنشاء العديد من الحاضنات التي تعتمد على التقنيات الناشئة الحديثة. وعلى الرغم من الجهود السابق ذكرها ترى العديد من الدراسات أن مصر جاءت في في

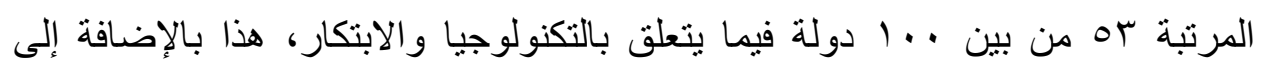
الإنفاق الضعيف على البحث والتطوير والمكانة الضعيفة للغاية في القدرات الاستيعابية للشركات. أما فيما يخص رأس المال البشري فقد جاءت في المرتبة 10 من بين . . 1. دولة، وذلك بسبب ظهور نقاط ضعف في القوى العاملة فيما يتعلق بالمهار ات ذات الصلة بالثورة الصناعية الرابعة، كما تحسن ترتيب مصر في الابتكار 9 مراتب بين عامي

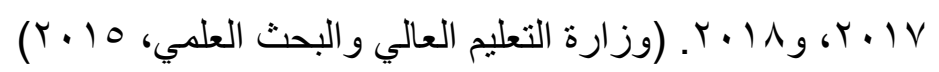
(Utikal et. al, 2019, 14) 
الثورة الصناعية الرابعة ومتطلبات تحقيقها في الجامعات المصرية

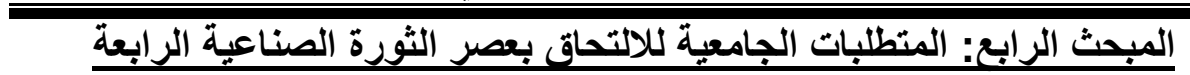
نظراً لأن الجامعات يقع على عاتقها مسئولية التنبؤ بالوظائف التي يتطلبها سوق العمل في المستقبل، وذلك من أجل تزويد المجتمع بخريجين لديهم القدرة على الالتحاق بهذه الوظائف. فمن الطبيعي أن يتمثل دور ها في تطوير مهار ات الطلاب لجعلهم قادرين على ادر اج واستثمار التقنيات الناثئة في سوق العمل، وهذا يتطلب إعادة هيكلة القوى البشرية للمؤسسة الجامعية وتطويرها من خلال تغيير أفكار الطلاب بما يتناسب مع احتياجات العصر، ومما ينعكس بطبيعة الحال على دور هم بعد التخرج، وبذلك يقع على بلى عاتقها بعض المتطلبات اللازمة لمو اكبة الثورة الصناعية الرابعة، والتي تتمثل في: ( ) قيادات ريادية

يوجد للقيادات الريادية الجامعية بالتعاون مع الحكومات دور فعال في تحقيق متطلبات الثورة الصناعية الرابعة بالجامعات والذي يتضح في الاهتمام بالبنية التحتية للتعليم والتدريب المهني للطلاب و أعضاء هيئة التدريس، من خلال وضع البنية التحتية و الأسس التنظيمية لنظام التعلم مدى الحياة من خلال ضمان جودة برامج التعليم، وضع المناهج والمعايير والأطر للاعتراف بالمهارات، و واستخدام البنية التحتية الثاسعة مدادة

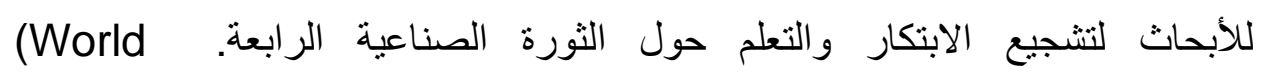
(Jadhav \& Mahadeokar, 2019, 107) Economic Forum a, 2017, 4) علاوة على تعزيز المساواة في الوصول من خلال ضمان الوصول إلى برامج التعليم الأساسي للكبار ذوي المهار ات المتدنية، تأمين الوصول إلى تقنيات التعلم، إنشاء

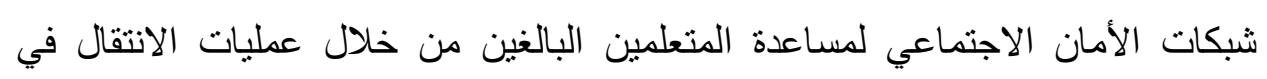

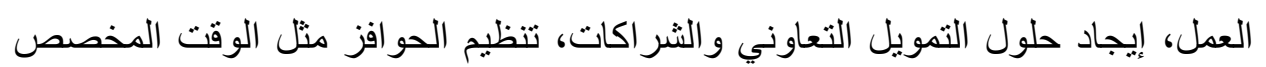

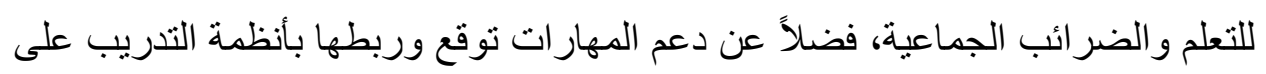
المستويين الوطني و المحلي. (World Economic Forum a, 2017, 4) 
أ/ هبة سمير سليمان محمود الجندى

r

يُعد التحول الرقمي من المتطلبات الأساسية للثورة الصناعية الرابعة الناتج عن استثمار تقنياتها الأساسية في المجتمع، حيث يتطلب هذا العصر ليتحول إلى مجتمع رقمي في الصناعة والتجارة والاقتصاد وسوق العمل، ما يلي: \& \&adhav) (القمة العالمية للصناعة والتصنيع (GMIS) Mahadeokar, 2019, 106) $(T \cdot r \cdot 17 \cdot(P W C) \&$

التكامل الأفقي والرأسي لسلاسل القيمة: رأسياً عبر المؤسسة بأكملها، بدءًا من تطوير المنتج وشر ائه، وحتى التصنيع و الخدمات، أما الأفقي يمتد إلى ما وراء العمليات الداخلية من الموردين إلى العملاء وجميع شركاء سلسلة القيمة الرئيسة، ويشمل جميع التقنيات من أجهزة التتبع والتعقب، وعمليات التخطيط المتكامل في الوقت الفعلي مع التنفيذ. ب) المنتجات والخدمات الرقمية : وذلك عن طريق إضافة أجهزة استشعار ذكية أو أجهزة اتصال يمكن استخدامها مع أدوات تحليل البيانات، وكذلك إنشاء منتجات رقمية جديدة تركز على حلول متكاملة، وجعل المؤسسات قادرة على توليد بيانات عن استخدام المنتج وتحسين المنتجات. ج) نماذج أعمال رقمية: من خلال توفير حلول رقمية مثل خدمات مبنية على البيانات، وحلول منصات متكاملة، وتوليد إير ادات رقمية إضافية وتحسين تفاعل العملاء. r) الموارد البشرية المؤهلة: وهذه الموارد تنقسم إلى أعضاء هيئة تدريس وطلاب لايهم مميزات خاصة وسوف يتم رصدها على النحو التالي: أ) (Lawrence et. al, 2019, عضو هيئة تدريس: (Ismail et al, 2020, 4) (20) 515- 516) (Sakhapov \& Absalyamova, 2018, 4) (Satpathy et. al, 2020, 2) 
الثورة الصناعية الرابعة ومتطلبات تحقيقها في الجامعات المصرية ومن اهم مميز ات عضو هيئة التدريس مايلي :

- - يكون مستعد لقبول التغييرات والابتكارات في نظام التعليم، أي أن يكون مستعد نفسيًا واجتماعيًا لمو اجهة تحديات هذه الثورة، لكئر - - يستطيع التعامل مع التغييرات والابتكارات التكنولوجية , بالإضافة إلى الاتصالات الحديثة،

- - يكون ديناميكيًا وقادرًا على التأقلم مع التغييرات المختلفة لتنويع أساليب التدريس و التعلم. - - يكون أكثر مسؤولية في إعداد خريجي اليوم بشكل أفضل لعالم القرن الحادي والعشرين الذي يتطلب تعريضهم للتكنولوجيا،

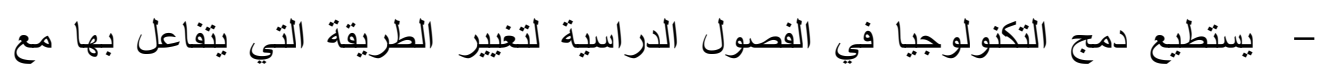

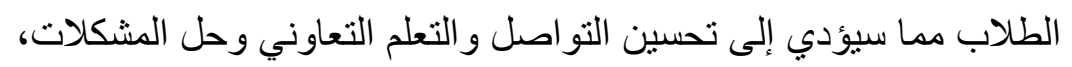

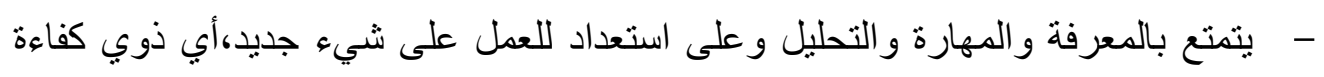

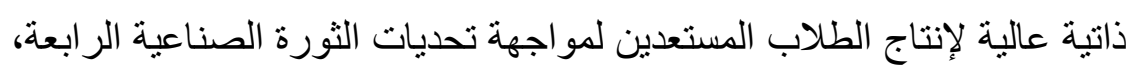

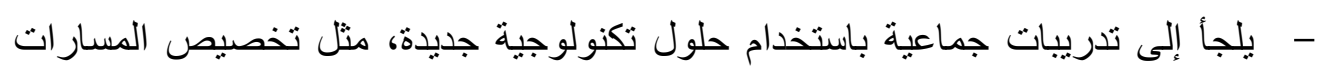

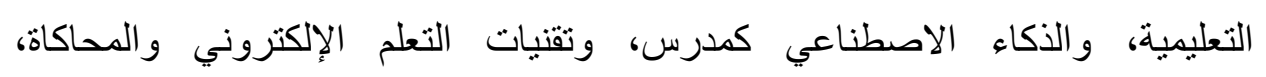

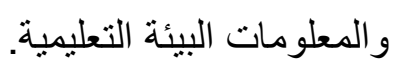

ب) الطلاب: بنطلب منها ما يلي: (Penprase, 2018, 223, 223 (225) (Shahroom \& Hussin, 2018, 317)

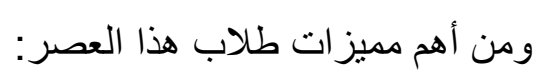
- - تحديث مهار اتهم بعد التخرج و إعادة الاتصال مع الخريجين الأكبر سنًا في بيئات الحرم

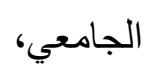
- التو اصل باستمرار مع زملائهم والخبر اء الخارجيين لتجديد وتحديث مهار اتهم،

مجلة كلية التربية- جامعة عين شثس 
أ/ هبة سمير سليمان محمود الجندى

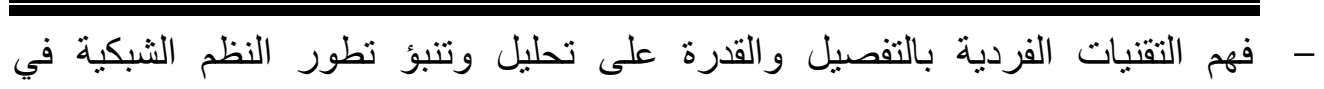
التكنولوجيا و البيئة والأنظمة الاجتماعية والسياسية، - - القدرة على التفكير الأخلاقي، و الوعي بالآثار الاجتماعية والبشرية. التي ستقوم بإعداد كل من الطلاب وأعضاء هيئة التدريس لأدوار قيادية في عالم يتسم بالتغير السريع المتسارع، (s) ع) التعليم الرقمي: يحاول الجزء الحالي التطرق للمتطلبات الخاصة واللازمة للتعليم القائم على تقنيات الثورة الصناعية الرابعة، و التي يتم رصدها على النحو التالي: البرامج الأكاديمية: ويجب أن ترتكز هذه البرامج في عصر الثورة الصناعية الرابعة

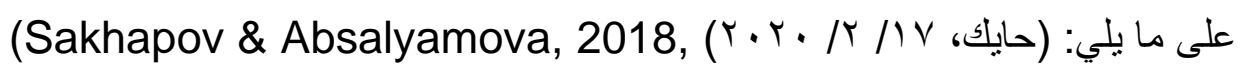

4) (Lawrence et. al, 2019, 511) - اثتراك العديد من أصحاب المصلحة أيضًا مع الجامعات لتطوير برامج تعليمية مخصصة لموظفيهم لإعدادهم لفرص العمل الناشئة، و التكيف مع بيئة العمل المتغيرة. - الاعتماد على برامج تركز على تطوير كفاءات معقدة متعددة التخصصات مرتبطة بالتفكير الإبداعي. - - تطوير برامج تعليمية جديدة لتلبية المتطلبات المتغيرة على المدى الطويل للحفاظ على ميزتها التنافسية. - إعادة التفكير في طريقة إعداد بر امج الدرجات العلمية، وحيث ير غب الطلاب في امتلاك خيارات حرة للاورات التعليمية، بعد الانتهاء من المساق الأكاديمي الذي تقدمه الجامعة، وذللك من أجل اكتساب مجموعة واسعة من المهارات. ب) تعليم ريادة الأعمال الرقمي الذي يعني دور الجامعات في بناء جيل يمنلك مهار ات ريادة

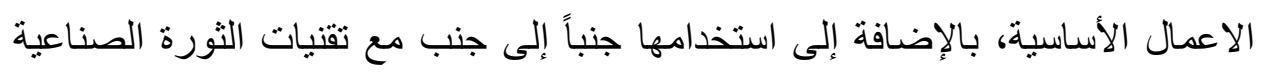

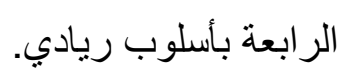

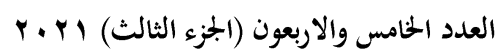

مجلة كلية التربية- جامعة عين شثس 
الثورة الصناعية الرابعة ومتطلبات تحقيقها في الجامعات المصرية

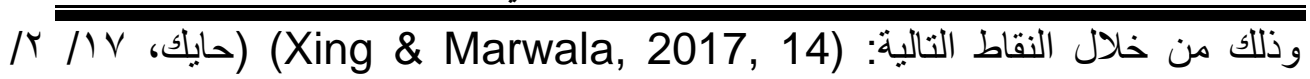
(Schwab, 2016,52) (Leurent \& (Naudé, 2017, 13: 17) (Y.r. (International Telecomunication Union, 2018, 6) Shook, 2019, 9) - - التحول نحو نقل المهارات المعقدة وحل المشكلات، أي الاعتماد على المهارات الإبداعية

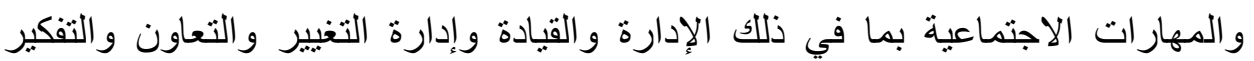
النقدي والفضول وأخذ المخاطر والتواصل و التنسويق و المبيعات. - - البدء في إعادة تدريب أعضاء هيئة التنريس على الانتقال إلى أساليب التدريس القائمة على حل الشكلات، و القائمة على المشرو عات. - تأمين الجامعات للتعليم الملائم للطلاب، وبما يضمن وجود فرصة لهم للعمل في

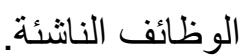

- زيادة الاستفادة من رواد الأعمال من قطاع الأعمال و العلوم في أنثطة التوعية التعليمية مثل الإعلان والبر امج عبر الإنترنت و التنلفزيون. - الاستعانة بالقادة المبنكرون و هذا يعني جلب تفكير جديد وإجراءات مختلفة لكيفية قيادة

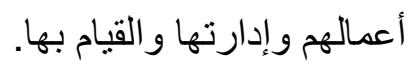
- - تهيئة البيئة و الثقافة لإطلاق العنان لابتكارات الآخرين في الجامعة التي تعزز تتوع

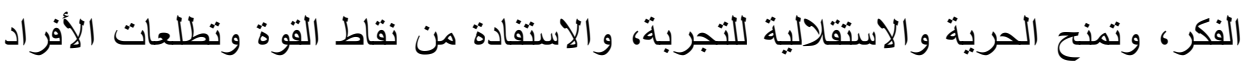

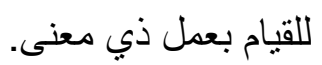
- تدريب الطلاب على مهارات ريادة الأعمال الرقمية، التي تجمع بين ريادة الأعمال التقليدية مع التقنيات الرقمية الجديدة، حيث تثميز المؤسسات الرقمية باستخدام التقنيات

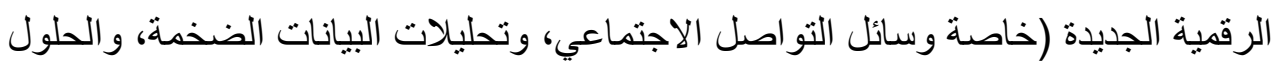

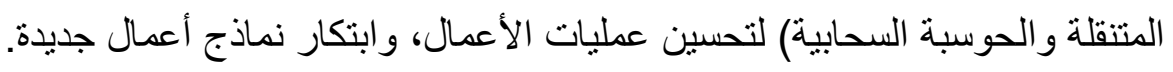




\section{أ/ هبة سمير سليمان محمود الجندى}

ج) المهارات: ونتيجة فقدان وظائف وظهور غير ها من الوظائف تتغير القوى العاملة المؤهلة وبطبيعة الحال تتطلب هذه التغييرات مهارات معينة لمواكبة سوق العمل، وفي وني هذا الجزء سوف تتطرق الدراسة لأهم المهارات الواجب اكتسابها في عصر الثورة الصناعية الر ابعة، وفقاً للنقاط التالية:

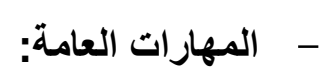

والتي تشمل المهارات النظرية التي يمكن أن يكتسبها الطلاب عبر التعليم الرسمي

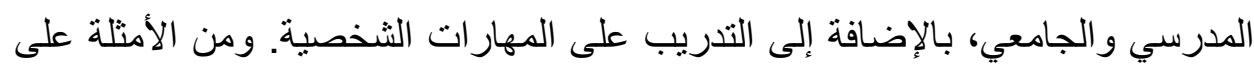

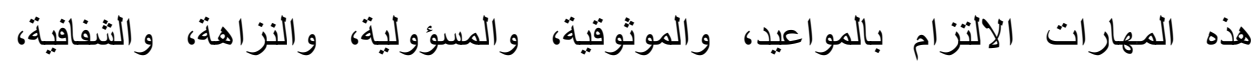
و الصدق، والقيم، والسلوكيات، والانضباط الذاتي، والثقة بالنفس، والمهار ات الاجتماعية

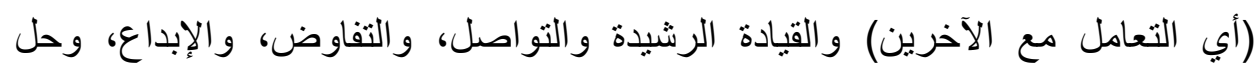
المشكلات. ومهارات الاتصال. (wadran, 2019, 12)

Forum, 2020, 12)

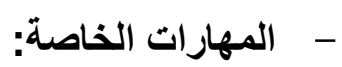
هي مجموعة من المهارات المتعلقة بمجال العمل، أي المهارات المطلوبة لبدء مشروع معين، فهي تتمحور حول مهار ات الصناعة المتخصصة بمجال المهن المعنية، و على هذا النحو فهي تتضمن مهارات مثل التسويق و إدارة المشاريع و الميز انية وإعداد

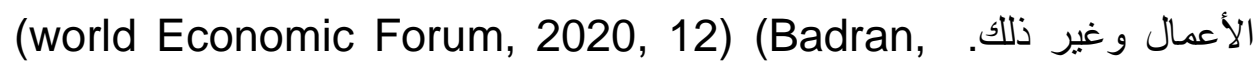
2019, 12)

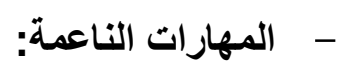
و هي تعني القدرة على التنقل بنجاح في مسارات العمل المختلفة من خلال العمل والأداء الجيد وذلك لتحقيق الأهداف المنشودة، فهذه المهار ات تعمل على تسليط الضوء على أهمية المهار ات الثخصية والسعي إلى فهها بشكل أعمق، وللمساعدة في ضمان حصول جميع الثباب على هذه المهار ات للتوظيف، ولتنمية هذه المهارات يجب التركيز 
الثورة الصناعية الرابعة ومنطلبات تحقيقها في الجامعات المصرية

على خمس مجموعات من المهارات: مفهوم الذات الإيجابي، والتحكم في النفس، و التواصل، والمهار ات الاجتماعية والتفكير العالي (الذي يشمل حل المشكلات و التفكير

(النقدي وصنع القرار) (Ignatowski, 2017, 4)

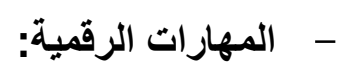

فهي تعني محو الأمية الرقمية أي تزويد الأفراد بمهارات تكنولوجيا المعلومات

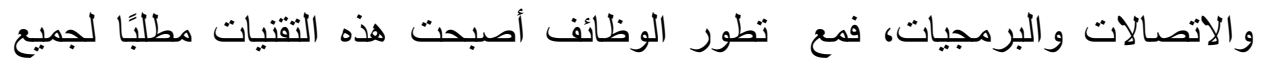
العمال بهدف التفاعل بين الإنسان والآلة، وبذانلك فهي تسمح للأفر اد باستخدام وتصميم

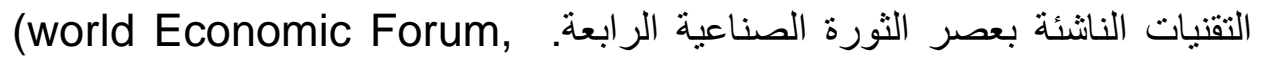

(Badran, 2019, 12) (Carter, 2019,18) 2020, 12) وبذلك فإن هذه المهارات تُعد أمراً ضروريًا لتزويد الداخلين إلى سوق العمل

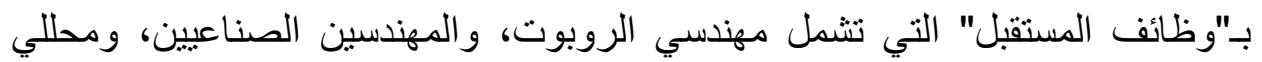
البيانات، والمهندسين المعماريين السحابيين، ومطوري البرمجيات، ومحللي الأمن.

(Naudé, 2017, 15)

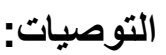

- تزويد أعضاء هيئة التدريس بمعلومات حول كيفية استخدام تقنيات الثورة الصناعية الر ابعة في العملية التعليمية.

- - نشر الوعي المجتمي بأهمية الثورة الصناعية الرابعة وماهيتها ومتطلباتها الجامعية. - - تشكيل فريق متخصص لدراسة التجارب العالمية التي قامت بتطبيق تقنيات الثورة

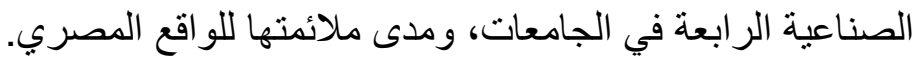

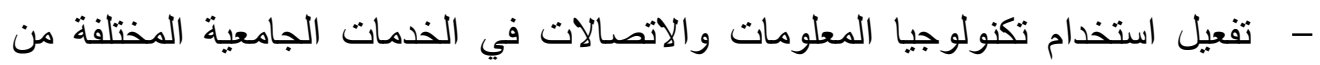
خلال تحويل المعلومات بالجامعات إلى محتوى رقات رقمي.

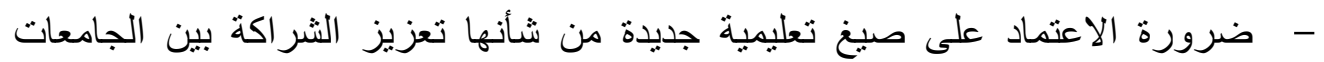
ومخرجاتها التعليمية. - - تعزيز الشر اكة بين المخرجات البحثية بالجامعات المختلفة و المؤسسات التنموية. - - توعية الطلاب بضرورة اكتساب المهارات اللازمة لسوق العمل المستقبلي.

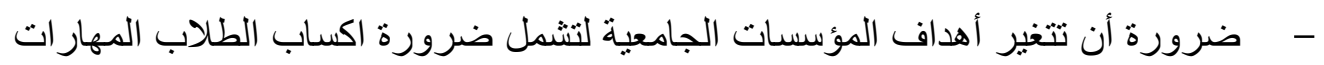

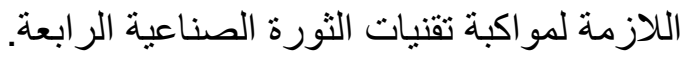

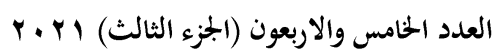

(196)

جلة كلية التربية- جامعة عين شمس 


\section{المراجع}

\section{أولاً: المراجع العربية}

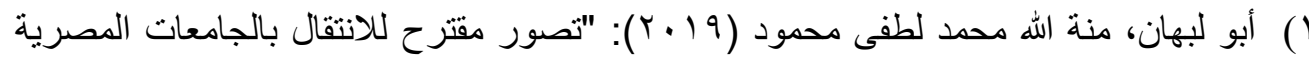

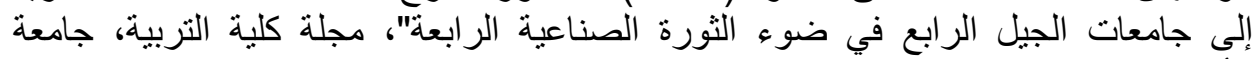

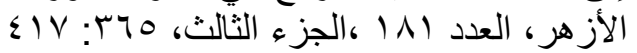

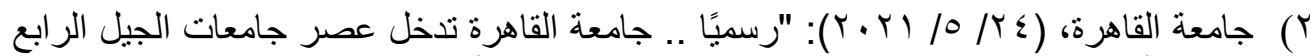

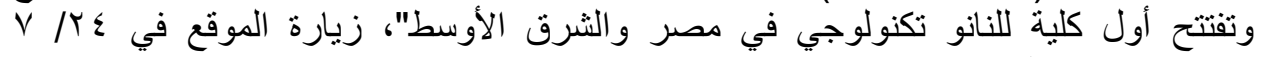

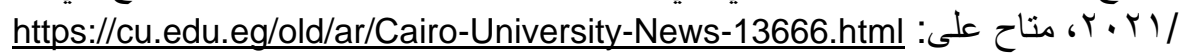

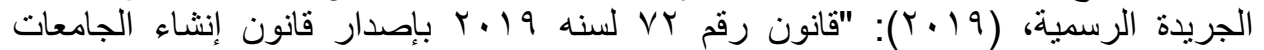

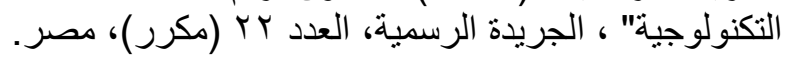

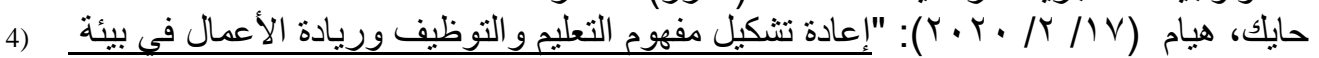

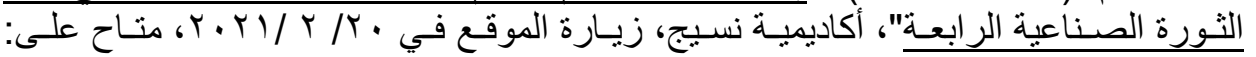
http://blog.naseej.com/\%D8\%A5\%D8\%B9\%D8\%A7\%D8\%AF\%D8\%A9-

\%D8\%AA\%D8\%B4\%D9\%83\%D9\%8A\%D9\%84-\%D9\%85\%D9\%81\%D9\%87\%D9\%88\%D9\%85\%D8\%A7\%D9\%84\%D8\%AA\%D8\%B9\%D9\%84\%D9\%8A\%D9\%85-

\%D9\%88\%D8\%A7\%D9\%84\%D8\%AA\%D9\%88\%D8\%B8\%D9\%8A\%D9\%81-

\%D9\%88\%D8\%B1\%D9\%8A\%D8\%A7\%D8\%AF\%D8\%A9-

\%D8\%A7\%D9\%84\%D8\%A3\%D8\%B9\%D9\%85\%D8\%A7\%D9\%84-\%D9\%81\%D9\%8A-

\%D8\%A8\%D9\%8A\%D8\%A6\%D8\%A9-\%D8\%A7\%D9\%84\%D8\%AB\%D9\%88\%D8\%B1\%D8\%A9\%D8\%A7\%D9\%84\%D8\%B5\%D9\%86\%D8\%A7\%D8\%B9\%D9\%8A\%D8\%A9\%D8\%A7\%D9\%84\%D8\%B1\%D8\%A7\%D8\%A8\%D8\%B9\%D8\%A9

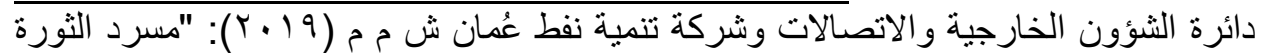

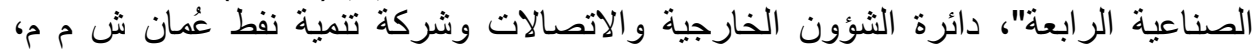

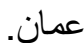
القمة العالمية للصناعة والتصنيع (GMIS) \& (PWC) (2016) (GM) الثورة الصناعية الرابعة:

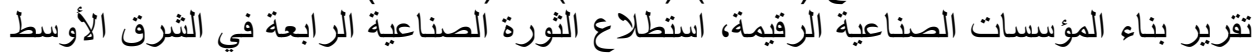

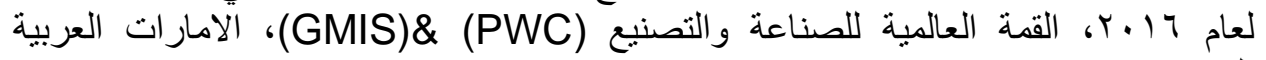

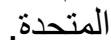

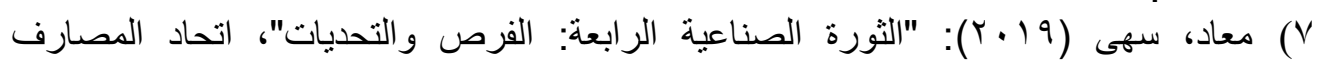
العربية، بيروت.

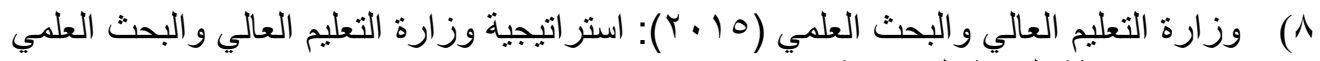

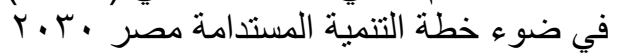

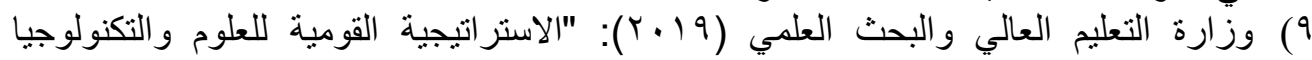

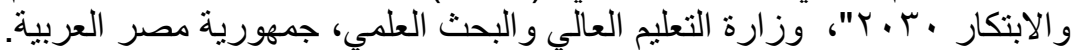

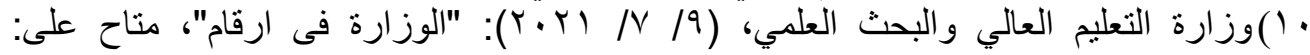
http://portal.mohesr.gov.eg/ar-eg/Pages/Facts figures.aspx ثانياً: المراجع الأجنبية 
1) Abbasy, Majid Bayani \& Quesada, Enrique Vílchez (2017): "Predictable Influence of IoT (Internet of Things) in the Higher Education", International Journal of Information and Education Technology, Vol. 7, No. 12, 914: 920

2) Abdel Ghaffar, Khaled (2020): "Harnessing rapid technological change for inclusive and sustainable development", United Nations Commission On Science and Technology for Development (CSTD), twenty-third session (virtual meeting) Geneva, 10-12 June 2020, Discussion on "Harnessing rapid technological change for inclusive and sustainable development", Egypt, Available at: https://unctad.org/system/files/non-officialdocument/ecn162020_s02_rapidtech_Egypt_KGhaffar_en.pdf

3) Asghar, Sohail et. al (2020): " The Fourth Industrial Revolution in the Developing Nations: Challenges and Road Map", Research Paper, Commission on Science and Technology for Sustainable Development in the South (COMSATS), Switzerland.

4) Badran, Mona Farid (2019): "Technological Change and its Impact on the Labor Market in Egypt, 2nd Europe - Middle East - North African Regional Conference of the International Telecommunications Society (ITS): "Leveraging Technologies For Growth", Aswan, Egypt, 18th-21st February, 2019, International Telecommunications Society (ITS), Calgary.

5) Benešová, Andrea \& Tupa, Jiří (2017): "Requirements for Education and Qualification of People in Industry 4.0", 27th International Conference on Flexible Automation and Intelligent Manufacturing, FAIM2017, 27-30 June 2017, Modena, Italy, Procedia Manufacturing, Vol 11, 2195 - 2202.

6) Blanchet, Max et. al (2014): "Industry 4.0 The new industrial revolution How Europe will succeed", Ronald Berger Strategy Consultants GMBH, Operations Strategy Competence Center (OPSCC), Germany.

7) Bryan, A. \& Volchenkova, K.N. (2018): "Blended Learning: Definition, Models, Implications for Higher Education", Bulletin of the South Ural State University. Ser. Education. Educational Sciences, vol. 8, no. 2, 24: 30

8) Carter, William (2019): "Defining the Technologies of the Fourth Industrial Revolution" In "Beyond Technology The Fourth Industrial Revolution in the Developing World", Aaron Milner and Erol Yayboke (Editors), A Report of the CSIS Project on Prosperity and development, Center for Strategic and International Studies (CSIS), Washington, D.C.

9) Challenor, Jennifer \& Ma, Minhua (2019): "A Review of Augmented Reality Applications for History Education and Heritage Visualisation", Multimodal Technol. Interact, Vol. 3, No. 39, 1: 20

10) Common Fund for Commodities (2018): “The Fourth Industrial Revolution: benefits and threats for commoditydependent developing countries", Common Fund for Commodities, Chipsafer, KIT royal trapical Institute.

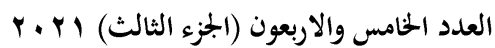

مجلة كلية التربية- جامعة عين شمس 
11) Cox, Joanna (2018): "The challenges posed and opportunities presented by the Fourth Industrial Revolution", the Institution of Engineering and Technology (The IET), Available at: https://www.theiet.org/media/2289/sub1061.pdf

12) Davies, Ron (2015): "Industry 4.0 Digitalisation for productivity and growth", European Parliamentary Research Service (EPRS), European Union.

13) Fahimirad, Mehrnaz \& Kotamjani, Sedigheh Shakib (2018): "A Review on Application of Artificial Intelligence in Teaching and Learning in Educational Contexts", International Journal of Learning and Development, Vol. 8, No. 4, 106: 118

14) Federal Ministry of Labour and Social Affairs (2017): "Re-imagining WorkWhite paperwork 0.4", Federal Ministry of Labour and Social Affairs, Directorate-General for Basic Issues of the Social State, the Working World and the Social Mark et Economy, Rostock.

15) Formunyam, Kahdinga George (2019): "Education and the Fourth Industrial revolution: Challenges and possibilities for Engineering Education", International Journal of Mechanical Engineering Technology (IJMET), Vol. (10), Issue (08), 271: 284.

16) Gueye, Mamadou \& Exposito, Ernesto (2020): "University 4.0: The Industry 4.0 paradigm applied to Education". IX Congreso Nacional de Tecnologías en la Educación, Oct 2020, Puebla (Mexico), France. ffhal02957371

17) Herweijer, Celine \& Waughray, Dominic (2018): "Fourth Industrial Revolution For the earth Harnessing Artificial Intelligence for the earth", PwC, UK.

18) Ignatowski, Clare (2017): "What Works in Soft Skills Development for Youth Employment? A Donors' Perspective, the Youth Employment Funders Group (YEFG) in partnership with the Mastercard Foundation.

19) International Telecommunication Union (2018): "Digital Skills Toolkit", International Telecommunication Union, Switzerland, Geneva.

20) Ismail, Nor Asmawati et al, (2020): "The Challenges of Industrial Revolution (IR) 4.0 towards the Teacher's Self-Efficacy", Journal of Physics: Conference Series, 1529, 042062, 1: 6

21) Jadhav, Viraj Vijay \& Mahadeokar, Ravindra (2019): "The Fourth Industrial Revolution (I4.0) in India: Challenges \& Opportunities", at Conference Issue Fostering Innovation, Integration and Inclusion Through Interdisciplinary Practices in Management, International Journal of Trend in Scientific Research and Development (IJTSRD), India, 105: 109

22) Kim, Sang Yun (2019): "The Fourth Industrial Revolution: Trends and Impacts on the World of Work", Springer Nature Switzerland, Switzerland.

23) Koziol, Maggie et. al, (2018): "Preparing tomorrow's workforce for the Fourth Industrial Revolution For business: A framework for action Executive summary", Deloitte Global and The Global Business Coalition for Education, Johannesburg

العدد الخامس والاربعون (الجزء الثالث) I r. r r

مجلة كلية التربية- جامعة عين شمس 


\section{الثورة الصناعية الرابعة ومتطلبات تحقيقها في الجامعات المصرية}

24) Krisnawati, Devi et. al, (2019): "Development Strategy of Study Programs in Higher Education to Respond the Fourth Industrial Revolution: Swot Analysis", RJOAS, Vol. 1, No. 85, 53: 61, Available at: https://rjoas.com/issue-201901/article_06.pdf

25) Lapteva, Alla V. \& Efimov, Valerii S. (2016): "New Generation of Universities. University 4.0", Journal of Siberian Federal University. Humanities \& Social Sciences, Vol 11, 2681-2696.

26) Lawrence, Rasika et al (2019): "Strengths and Weaknesses of Education 4.0 in the Higher Education Institution", International Journal of Innovative Technology and Exploring Engineering (IJITEE), ISSN: 2278-3075, Volume-9 Issue-2S3, pp 511: 512

27) Leurent, Helena \& Shook, Ellyn (2019): "Leading through the Fourth Industrial Revolution Putting People at the Centre In collaboration with Accenture", White paper, World economic forum.

28) Manda, More Ickson \& Dhaou, Soumaya Ben (2019): "Responding to the challenges and opportunities in the $4^{\text {th }}$ Industrial revolution in developing countries", Association for Computing Machinery, April 3-5, Melbourne, VIC, Australia.

29) McKinsey Global Institute (2015): “THE INTERNET OF THINGS: MAPPING THE VALUE BEYOND THE HYPE, EXECUTIVE SUMMARY, McKinsey Global Institute

30) Munnerley, Danny et. al, (2014): "Augmented Reality: application in Higher Education", Final Report 2014, Australian Government, Ofiice for Learning and Teaching.

31) Naudé, Wim (2017): "Entrepreneurship, Education and the Fourth Industrial Revolution in Africa", Discussion Paper Series, IZA- Institute of labor Economics, bonn, Germany.

32) Nkosi, Thalente et al (2020): "Harnessing Fourth Industrial Revolution(4IR) for Improving Poor Universities Infrastructure in Developing Countries-A Review", Proceedings of the International Conference on Industrial Engineering and Operations Management Dubai, UAE, March 10-12, 2020, IEOM Society International, 2114; 2125.

33) Penprase, Bryan Edward (2018): "The Fourth Industrial Revolution and Higher Education", the registered company Springer Nature Singapore Pte Ltd, Singapore

34) Quyet, Nguyen Thi (2020): "Higher Education in the Fourth Industrial Revolution Age", American Journal of Educational Research, Vol. 8, No. 6, 420: 426.

35) Rizov, Tashko \& Rizova, Elena (2015): "Augmented Reality as a Teaching Tool in Higher Education", (IJCRSEE) International Journal of Cognitive Research in Science, Engineering and Education Vol. 3, No.1, 7: 16

العدد الخامس والاربعون (الجزء الثالث) ا Y r r

$(200)$
مجلة كلية التربية- جامعة عين شمس 
36) sakhapov, Rustem \& Absalyamova, Svetlana (2018): "Fourth industrial revolution and the paradigm change in engineering education", EDP sciences, Russia.

37) Satpathy, Swayamprabha et al (2020): "A Study on the New Design Thinking for Industrial Revolution 4.0, Requirements and Graduate Readiness", Rupkatha Journal on Interdisciplinary Studies in Humanities, Vol. 12, No. 4, 1: 11

38) Schwab, Klaus (2016): "The Fourth Industrial Revolution", World Economic Forum, Switzerland.

39) Shahroom, Aida Aryani \& Hussin, Norhayati (2018): "Industrial Revolution 4.0 and Education", International Journal of Academic Research in Business and Social Sciences, Vol. 8, No. 9, 314: 319

40) Sural, Irfan (2017): " Mobile Augmented Reality Applications in Education", In "Mobile Technologiesand Augmented Reality in Open Education", IGI Global, United States.

41) Thang, Le Van \& Dung, Nghiem Xuan (2018): "Building the Higher Education 4.0 In the Armed Forces Associated with The Industry 4.0: Potential and Challenges", Journal of Interdisciplinary Research, ADALTA, 171: 175

42) The Alcatel-Lucent, (2020): "The Internet of Things in Education Improve learning and teaching experiences by leveraging IoT on a secure foundation", Solution brief IOT in Education, Available at: https://www.al-enterprise.com/Imedia/assets/internet/documents/iot-for-education-solutionbrief-en.pdf

43) The National Science and Technology Council (NSTC), The Office of Science and Technology Policy (OSTP), (2016): "Preparing for The Future of Artificial Intelligence", Executive Office of the President National Science and Technology Council Committee on Technology.

44) The United Nations Educational, Scientific and Cultural Organization, (2019): "Artificial Intelligence in Education: Challenges and Opportunities for Sustainable Development", Working Papers on Education Policy, the United Nations Educational, Scientific and Cultural Organization, France.

45) Um, Jung-Sup (2019): "Drones as Cyber-Physical Systems Concepts and Applications for the Fourth Industrial Revolution", Springer Nature Singapore, Singapore

46) Utikal, Hannes et al (2019): "Industry 4.0 in Sustainable Industrial Areas in Emerging and Developing Countries", Deutsche Gesellschaft für Internationale Zusammenarbeit (GIZ) GmbH", Eschborn.

47) World Economic Forum (2016): "The Future of Jobs Employment, Skills and Workforce Strategy for the Fourth Industrial Revolution", World Economic Forum, Geneva, Switzerland.

48) World Economic Forum (2020): "Jobs of TomorrowMapping Opportunity in the New Economy", World Economic Forum, Switzerland.

العدد الخامس والاربعون (الجزء الثالث) ا Y r r

(201)
جلة كلية التربية- جامعة عين شمس 


\section{الثورة الصناعية الرابعة ومتطلبات تحقيقها في الجامعات المصرية}

49) World Economic Forum a (2017): "Accelerating Workforce Reskilling for the Fourth Industrial Revolution an Agenda for Leaders to Shape the Future of Education, Gender and Work- White Paper", World Economic Forum, Switzerland.

50) World Economic Forum b (2017): "ASEAN 4.0: What does the Fourth Industrial Revolution mean for regional economic integration?" White paper, World Economic Forum, Switzerland.

51) Xing, Bo \& Marwala, Tshilidzi (2017): "Implications of the Fourth Industrial Age for Higher Education", Science and Technology, Vol. 73, 10: 15

52) $\mathrm{Xu}$, Min et.al, (2018): "The Fourth Industrial Revolution: Opportunities and Challenges", International Journal of Financial Research, Vol. 9, No. 2, 90: 95

53) Grinshkun, Vadim \& Osipovskaya, Elizaveta (2020): "Teaching in the Fourth Industrial Revolution: Transition to Education 4.0", Proceedings of the 4th International Conference on Informatization of Education and E-learning Methodology: Digital Technologies in Education (IEELM-DTE 2020), Krasnoyarsk, Russia, October 6-9.

54) Eleyyan, Shaher (2021):"The future of education according to the fourth industrial revolution", Journal of Educational Technology \& Online Learning, 4(1), 23-30.

55) Oke, Adekunle \& Fernandes, Fatima Araujo Pereira (2020): "Innovations in Teaching and Learning: Exploring the Perceptions of the Education Sector on the 4th Industrial Revolution (4IR)", Journal of open innovation: Technology, Market and Complexity, Vol 6, No. 31, 1: 22 